\title{
A benders-local branching algorithm for second-generation biodiesel supply chain network design under epistemic uncertainty
}

\author{
Reza Babazadeh ${ }^{\mathrm{a}, *}$, Hamid Ghaderi ${ }^{\mathrm{b}}$, Mir Saman Pishvaee ${ }^{\mathrm{b}}$ \\ ${ }^{a}$ Faculty of Engineering, Urmia University, Urmia, West Azerbaijan Province, Iran \\ ${ }^{b}$ School of Industrial Engineering, Iran University of Science and Technology, Tehran, Iran
}

\begin{abstract}
This paper proposes a possibilistic programming model in order to design a second-generation biodiesel supply chain network under epistemic uncertainty of input data. The developed model minimizes the total costs of the supply chain from supply centers to the biodiesel and glycerin consumer centers. Waste cooking oil and Jatropha plants, as non-edible feedstocks, are considered for biodiesel production. To cope with the epistemic uncertainty of the parameters, a credibility-based possibilistic programming approach is employed to convert the original possibilistic programming model into a crisp counterpart. An accelerated benders decomposition algorithm using efficient acceleration mechanisms is devised to deal with the computational complexity of solving the proposed model in an efficient manner. The performance of the proposed possibilistic programming model and the efficiency of the developed accelerated benders decomposition algorithm are validated by performing a computational analysis using a real case study in Iran.
\end{abstract}

Keywords: Bioenergy, Biofuel supply chain, Optimization, Benders Decomposition, Uncertainty.

\section{Introduction}

In the last decades, mounting concerns about energy security, environmental issues, and food supply have resulted in searching for renewable and cleaner energy resources. Transportation sector has a substantial share in energy demand in comparison with other sectors demanding energy. Researchers and policymakers have introduced the utilization of biofuels so as to response to the serious need for renewable and cleaner energy in transportation sector. China has established more than 2000 biodiesel factories by 2007 and United States aims to 36 billion gallons of biofuels by 2022 [1].

Biodiesel is a kind of biofuel which can be successfully combined with fossil-based diesel in different percentages in order to use in transportation sector. Biodiesel production from first generation biomass has been commercialized at worldwide. However, Biodiesel production from first generation biomass is not sustainable inasmuch as it seriously aggravates food crisis. Hence, recently, the researchers and policymakers have introduced the biomass feedstocks whose required land for cultivation cannot be applied

* Corresponding author. Tel.: +98 44 32972854; Fax: +98 4432773591.

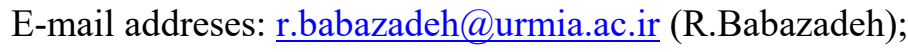


to produce crop; rather, they enjoy all the features that are required for producing biodiesel. Jatropha Curcas L. (JCL) has been known as the most auspicious non-edible energy crop for second-generation biodiesel production $[2,3]$. JCL seed kernel has about $30-40 \%$ oil and its oil content is ranked second among edible and non-edible energy crops [2]. The considerable advantages of JCL include growth in marginal lands, soil reclamation, prevention of soil erosion, improvement in water infiltration, being drought tolerant, low water and nutrient requirement, greenhouse gas emission reduction, and contribution to the rural development [2, 3]. Waste Cooking Oil (WCO) has been known as another non-edible source of producing biodiesel that helps feasibility of biodiesel production and improves its competition with fossil-based diesel. Moreover, a suitable planning for WCO collection helps to resolve its associated disposal issues and provides a very low price feedstock for biodiesel production [4].

In literature on the subject of Biofuel Supply Chain Network Design (BSCND) problem, various approached have been applied. These approaches are classified into three groups, including (1) mathematical programming, (2) multi-criteria decision-making, and (3) heuristic or metaheuristic approaches, among which mathematical programming is the most frequently-used approach [5]. Given the objective function, constraints, and decision variables, mathematical programming models can be categorized into three groups, including (1) linear programming (e.g. [6, 7]), (2) mixed integer linear programming (e.g. [4, 8-10]), and (3) nonlinear programming (e.g. [11, 12]). Multi-criteria decision-making methods are classified into two groups, including (1) multi-attribute decision-making methods [13] and (2) multi-objective decision-making methods (e.g. [10, 14, 15]). Heuristic and metaheuristic algorithms (e.g. [16]) can be used for solving problems that have high computational complexity and reaching near optimal solutions in a short period of time. For more details about the approaches that have been used in BSCND problems, interested readers may consult with Ghaderi et al. [5] that provides the readers with a comprehensive review on BSCND models.

Due to the dynamic environment under which a biofuel supply chain operates, a considerable degree of uncertainty affects the strategic decisions [5]. In general, supply chain network design models will not be reliable enough if the uncertainty of the problem is not handled. In BSCND models, some parameters such as biomass supply, biofuel production, demand, and prices can be under uncertainty [5]. The input data in a problem can be under two types of uncertainty, including: (1) randomness that arises from the random nature of events and addresses the uncertainty about the membership or non-membership of an element in a set. Accordingly, stochastic programming approaches can be used for modeling the randomness of the parameters and (2) epistemic uncertainty that is a source of non-deterministic behavior arises from lack of knowledge of the system or environment [17]. Indeed, the main source of epistemic uncertainty is incomplete information about the system, some examples of which are little or no experimental data for a parameter, a range of possible values of a parameter that are provided by expert opinions, limited understanding of complicated processes, and the existence of some environmental conditions or incorrect sequences that are not identified for inclusion in the system analysis [18]. It is worth mentioning that in most of the real world problems, there might be not sufficient historical data for modeling non-deterministic parameters as random data. As a result, such uncertain parameters are estimated according to the experts' judgments, which are mainly based on their experiences and professional opinions. With regard to this, possibilistic programming approaches can be applied to this type of uncertainty [19]. Therefore, the approach used for modeling the uncertainty of BSCND problems can be different regarding the type of uncertainty. In most of the research in the literature on the subject of BSCND (e.g. [20-22]), stochastic programming models have been developed to address the uncertainty of the problems. Nevertheless, we usually encounter incomplete information in the real world problems [23]. Thus, using the approaches that 
are capable of modeling the incomplete information is of great importance as far as BSCND is concerned. Regarding this, a number of researchers (e.g. [12, 24, 25]) have used possibilistic programming approaches in BSCND problems.

Motivated from the shortcomings in literature, in this paper, an MILP model is proposed to design a secondgeneration biodiesel supply chain network under epistemic uncertainty. The proposed model integrates all stages from supply centers to biodiesel and glycerin consumer centers. The developed model minimizes the total costs subject to real-life assumptions and constraints. Non-edible JCL seed oil and WCO are considered as the second-generation feedstocks for biodiesel production. The proposed model exclusively considers marginal lands for JCL cultivation, which leads to the avoidance of competition for fertile lands. The proposed model is developed under uncertainty of JCL seed oil and WCO supply, biodiesel demand, and cost coefficients. Due to lack of reliable historical data and therefore epistemic uncertainty of the parameters, a possibilistic programming model based on credibility measure is proposed for designing the given supply chain. To the best of our knowledge, this is the first time that credibility measure is used to model uncertainty in a second-generation biodiesel supply chain network design problem. To solve the developed model, this paper proposes a strong Benders Decomposition Algorithm (BDA) that greatly benefits from a number of acceleration methods in order to considerably accelerate the convergence of the algorithm. However, the main limitation of this work is that the proposed approach could not deal with optimization problems with both randomness and epistemic uncertainty.

The rest of this paper is organized as follows. Literature review is presented in Section 2. The given problem is defined in Section 3. The developed model as well as method proposed to deal with uncertain parameters are illustrated in Section 4. The used solution approach is described in Section 5. The studied case and the results obtained from the proposed model and solution approach are presented in Section 6. Finally, Section 7 concludes this article and offers some research directions.

\section{Literature Review}

Despite the fact that uncertainty in a BSCND model makes it a more realistic and appropriate tool for energy policymakers, deterministic models are the most frequently-developed models in the literature [5]. Ahn et al. [26] developed a deterministic MILP model so as to design a microalgae to biodiesel supply chain network. Babazadeh et al. [4] developed a hybrid approach based on Data Envelopment analysis (DEA) and MILP methods to design a biodiesel supply chain and find the optimal number, location, and capacity of facilities and transportation modes. Paulo et al. [27] proposed an MILP model in order to design a forestry biomass to bioelectricity supply chain. Their proposed model finds the optimal capacity and location of facilities, the amount of biomass feedstock, transportation modes and the links among them. For strategic optimizing bioethanol and bioenergy supply chains, d'Amore and Bezzo [28] developed a multi-objective MILP which maximizes net present value and minimizes total greenhouse gas emissions of the supply chain. Incorporating all policy instruments of European biofuel regulations, Hombach et al. [29] developed an MILP model in order to design sustainable second-generation biofuel supply chains. In this model, maximizing net present value and maximizing greenhouse gas emission saving quotas are considered as the economic and environmental objective functions, respectively. In order to design sustainable biofuel supply chains, Yue et al. [30] presented multi-objective MILP models in which the maximization of costs, minimization of greenhouse gas emissions, and maximization of job opportunities are the objective 
functions. Given various impacts of new created jobs based on their type and location, Cambero and Sowlati [10] developed an indicator in order to quantify the potential social benefit of forest-based bioenergy supply chains. They incorporated this social benefit into a multi-objective MILP model so as to maximize the social benefit, net present value, and greenhouse gas emission potential saving of the supply chain. In order to determine the optimal design of a sustainable biofuel supply chain from coffee cut stem, Duarte et al. [31] proposed an MILP model in which a global objective function in terms of economic benefits and positive environmental balance is maximized.

A number of researchers have coped with the uncertainty of the parameters in BSCND models. Osmani and Zhang [22] developed a stochastic MILP model for optimizing sustainable biofuel supply chains. Zhang and Jiang [32] proposed a robust MILP model for the design of a sustainable WCO to biodiesel supply chain under price uncertainty. Ghaderi et al. [33] developed an MILP model so as to design a switchgrassbased bioethanol supply chain in which bioethanol demand changes under auto-regressive moving average time series models. Mohseni et al. [9] developed a two-stage model in order to design and plan a microalgaebased biodiesel supply chain. In this model, the macro-stage conducts a spatial filtering through GIS and AHP to determine the best locations for establishing biodiesel production facilities. In the micro-stage, a robust MILP model that makes a trade-off between supply chain total costs and reliability is presented in order to find the value of strategic and tactical decision variables that are optimal for almost all possible realization of uncertain parameters. Mohseni et al. [34] proposed a robust MILP model that find the optimal value of the microalgae-based biofuel supply chain decisions considering upper and lower bound of uncertain parameters. The formulation of the developed model is based on batch and continuous production systems that entirely cover the possible future configuration of the given supply chain and provides managerial implications that contribute to cost reduction.

Due to lack of reliable historical data and knowledge in a BSCND problem, a number of parameters may encounter epistemic uncertainty; hence, possibilistic programming models can be used to deal with such uncertain parameters. Regarding this, Tong et al. [24] proposed an MILP model for the design of a biofuel supply chain integrated with petroleum refineries. To model uncertainty based on decision makers' preferences, they developed a possibilistic programming approach in which possibility, necessity, and credibility measures are used. Babazadeh et al. [12] defined possibilistic mean value and absolute deviation of fuzzy numbers and developed new formulations of possibilistic programming and robust possibilistic programming in order to design a sustainable second-generation biodiesel supply chain. In order to design a sustainable bioethanol supply chain under multiple uncertainties, Bairamzadeh et al. [8] developed a multi-objective robust possibilistic programming method to cope with the uncertainty of the parameters. Ghaderi et al. [25] developed a multi-objective robust possibilistic programming model for designing a sustainable switchgrass-based bioethanol supply chain network under epistemic uncertainty. Their proposed model is capable of maximizing the mean value of supply chain performance, optimality robustness and feasibility robustness. Bairamzadeh et al. [35] developed a hybrid robust optimization model in which conversion rate is expressed as probabilistic scenarios; biomass yield is presented as fuzzy numbers, and demand changes in a known interval. Potential disruptions such as flood, drought, pest, and equipment failure occasionally occur in biofuel supply chains, therefore, these disruptions are supposed to be considered in BSCND. With regard to this, Maheshwari et al. [36] proposed a BSCND model that incorporates the possibility of disruption in designing the supply chain.

It is worth bearing in mind that using stochastic programming methods to address uncertainty of parameters requires reliable historical data. In addition, these methods intensify the computational complexity of BSCND problems. These disadvantages decrease the practicality of stochastic programming methods in 
real-world problems, where possibilistic programming methods overcome these disadvantages. Moreover, the literature lacks integrated models for designing second-generation biodiesel supply chains under real world assumptions and epistemic uncertainty of the parameters. To the best of our knowledge, there is not any research in the literature that uses credibility measure to model epistemic uncertainty in a secondgeneration biodiesel supply chain network design problem.

\section{Problem definition}

The given problem in this study is designing a second-generation biodiesel supply chain network that integrates all stages from supply centers to the biodiesel and glycerin consumer centers under epistemic uncertainty. The given problem is a multi-feedstock, multi-product, multi-period, multi-modal BSCND problem. Fig. 1 illustrates the structure of the given supply chain. As it can be seen in Fig. 1, JCL seeds are harvested from farms and transported to the JCL oil extraction centers. Then, the extracted oil is shipped to the bio-refineries. WCO is collected from supply centers and transported to the bio-refineries. At biorefineries, JCL oil and WCO are transformed into biodiesel and glycerin. Finally, the produced glycerin is shipped to glycerin consumers and the produced biodiesel is transported to the consumer centers and is blended with fossil-based diesel in a predetermined percentage. JCL cultivation is not dependent on time period and all needed JCL is cultivated in the first year. JCL trees yield after 2 years of cultivation. Also, JCL reaches full fruity after 5 years and remains at the fixed level of fruiting for 40 years. All the needed JCL is cultivated in the first year to be harvested in full fruity state in the upcoming periods. JCL farms and all facilities have capacity. Multiple capacity levels are considered for JCL farms and all facilities. The suitable capacity of facilities is determined according to the optimal structure of the supply chain. Oil extraction centers and bio-refineries are established with an initial capacity level. Then, in the next periods, their capacity can be expanded according to the increase in the consumers' demand. The established facilities will be active by the end of the planning horizon. Two transportation modes, rail and road, link all the stages of the supply chain, and it is assumed that transportation capacity is infinite. Additionally, each consumer center is assumed to be known and deterministic. The problem is considered for a 7-year planning horizon. Nevertheless, to decrease computational complexity of the problem, each year is regarded as a time period. Accordingly, the overall planning horizon includes seven time periods. It is assumed that JCL is cultivated only in arid and semi-arid lands rather than fertile areas. Also, inventory decisions are not considered in different levels of the biodiesel supply chain. The main decisions of the proposed model are determining the optimal sourcing and allocation of JCL seeds and WCO; the capacity and location of JCL supply centers, oil extraction centers, and bio-refineries; the capacity expansion of oil extraction centers and bio-refineries; the amount of imported JCL seeds to oil extraction centers; the amount of produced JCL oil at oil extraction centers; the amount of produced biodiesel and glycerin at bio-refineries; the material flow between the stages of the supply chain; and transportation modes in different periods so much so that the total costs of the supply chain is minimized. Due to lack of required historical data, probability distributions cannot be applied to this study. Hence, an appropriate substitute for probability distribution is required to formulate the uncertain parameters based on both available (but not sufficient) data and experts'

opinions. In this regard, trapezoidal possibility distributions are used, and a possibilistic programming model based on credibility measure is developed for the optimal design of given supply chain. 


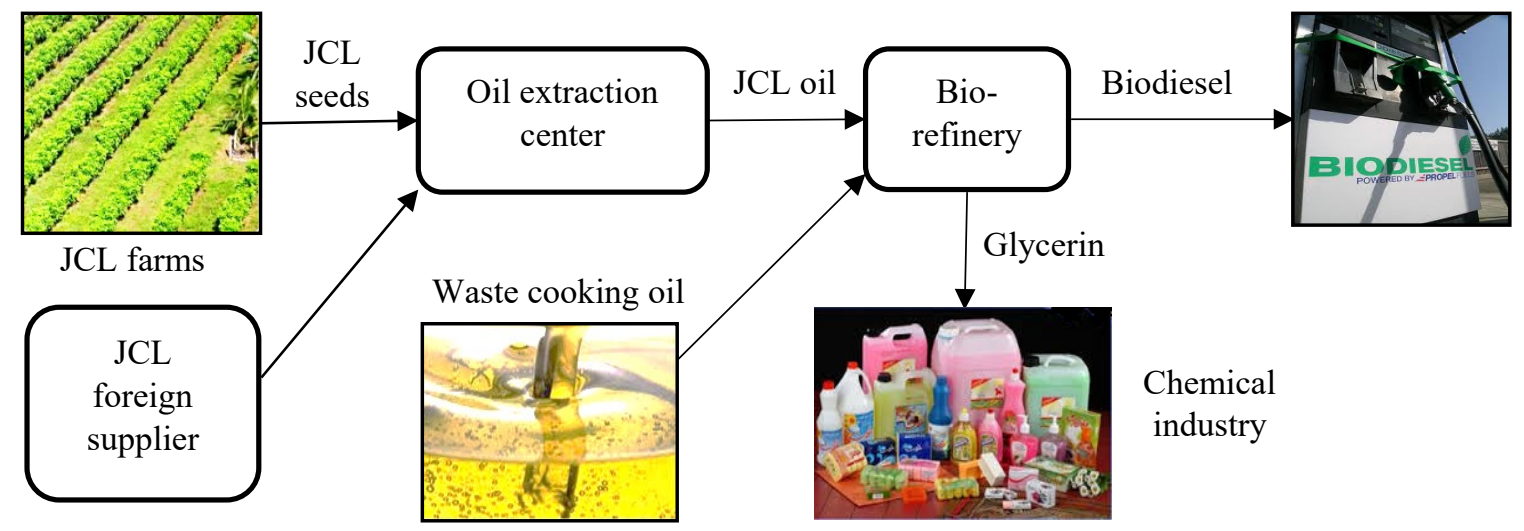

Fig. 1. Second-generation biodiesel supply chain structure

\section{Model formulation}

\subsection{Proposed mathematical model}

The indices, parameters, and variables of the proposed model are defined as follows. It should be noted that the parameters with a tide on show uncertain coefficients.

$\begin{array}{ll}\text { Indices } & \\ f & \text { Index of candidate locations for JCL supply centers } \\ g & \text { Index of candidate locations for WCO supply centers } \\ i & \text { Index of candidate locations oil extraction centers of JCL yields } \\ j & \text { Index of candidate locations for bio-refineries of biodiesel production } \\ l & \text { Index of transportation mode (road and railway) } \\ k & \text { Index of consumer centers of biodiesel } \\ p & \text { Index of the capacity levels of JCL supply centers } \\ q & \text { Index of the capacity levels of oil extraction centers of JCL yields } \\ s & \text { Index of the capacity levels of bio-refineries of biodiesel production } \\ t & \text { Index of time period } \\ \text { Technical and economic parameters } \\ \widetilde{D}_{k t} & \text { Demand of consumer center } k \text { for biodiesel in period } t \text { (ton/period) } \\ \widetilde{W} O_{g t} & \text { Amount of WCO supplied by supply center } g \text { in period } t \text { (ton/period) } \\ \tilde{\eta}_{f t} & \text { Amount of JCL yields per hectare at location } f \text { in period } t \text { (ton/ha) } \\ \varphi & \text { Conversion factor of JCL yields to JCL oil (percent) } \\ \beta & \text { Conversion factor of JCL oil or WCO to biodiesel } \\ L J_{f} & \text { Minimum land area dedicated for a JCL cultivation center at location } f \text { (ha*) } \\ U J_{f} & \text { Maximum land area available for a JCL cultivation center at location } f \text { (ha) } \\ L O_{i} & \text { Lower bound dedicated on capacity of an oil extraction center of JCL yields at location } i \\ U O_{i} & \text { (ton) }\end{array}$


$L B_{j} \quad$ Lower bound dedicated on capacity of a bio-refinery at location $j$ (ton)

$U B_{j} \quad$ Upper bound of capacity of a bio-refinery at location $j$ (ton)

Maxy Maximum number of locations which can be selected for opening oil extraction centers

Maxu Maximum number of locations which can be selected for opening bio-refineries

$M I_{i t} \quad$ Maximum amount of JCL seeds that could be imported to oil extraction center $i$ in period

$\widetilde{F J}_{f}^{p} \quad$ JCL cultivation cost at location $f$ with capacity level $p$ (MIRR*)

$\widetilde{F O}_{i t} \quad$ Fixed cost of opening an oil extraction center of JCL seeds at location $i$ in period $t$ (MIRR)

$\widetilde{F B}_{j t} \quad$ Fixed cost of opening a bio-refinery at location $j$ in period $t$ (MIRR)

$\widetilde{V O}_{i t}^{q} \quad$ Cost of capacity expansion equal to $q$ at oil extraction center $i$ in period $t$ (ton)

$\widetilde{V B}_{j t}^{S} \quad$ Cost of capacity expansion equal to $s$ at bio-refinery $j$ at period $t$ (ton)

$\widetilde{P O}_{i t} \quad$ Unit oil extraction cost from JCL seeds at oil extraction center $i$ in period $t$ (MIRR.ton ${ }^{-}$

$1 /$ period)

$\widetilde{P B}_{j t} \quad$ Unit production cost of biodiesel at bio-refinery $j$ in period $t$ (MIRR.ton ${ }^{-1} /$ period)

$\widetilde{J T}_{\text {flit }} \quad$ Transportation cost of JCL seeds from cultivation center $f$ to oil extraction center $i$ by mode $l$ in period $t$ (MIRR. ton $^{-1}$ period)

$\widetilde{O T}_{i l j t} \quad$ Transportation cost of JCL oil from oil extraction center $i$ to bio-refinery $j$ by model $l$ in period $t$ (MIRR.ton ${ }^{-1} /$ period)

$\widetilde{W T}_{g l j t} \quad$ Transportation cost of WCO from supply center $g$ to bio-refinery $j$ by mode $l$ in period $t$ (MIRR. ton $^{-1}$ period)

$\widetilde{B T}_{j l k t} \quad$ Transportation cost of biodiesel from bio-refinery $j$ to consumer center $k$ by

$\widetilde{P I}_{i t} \quad$ Importing cost of JCL seeds in oil extraction center $i$ in period $t$

$\widetilde{P G}_{j t} \quad$ Selling price of glycerin at bio-refinery $j$ in period $t$

$D i s J T_{f l i} \quad$ Distance between JCL supply center $f$ and oil extraction center $i$ by mode $l$

$D_{i s} O T_{i l j} \quad$ Distance between oil extraction center $i$ and bio-refinery $j$ by mode $l$

$D i s W T_{g l j} \quad$ Distance between WCO supply center $g$ and bio-refinery $j$ by mode $l$

$D i s B T_{j l k} \quad$ Distance between bio-refinery $j$ and consumer center $k$ by mode $l$

$C J_{f}^{p} \quad$ Amount of cultivated area in JCL supply center $f$ with capacity level $p$ (ha)

$I \mathrm{CO}_{i t} \quad$ Initial capacity of oil extraction center $i$ that is established in period $t$

$I C B_{j t} \quad$ Initial capacity of bio-refinery $j$ established in period $t$

$C E O_{i t}^{q} \quad$ Amount of capacity expansion $q$ in oil extraction center $i$ in period $t$

$C E B_{j t}^{S} \quad$ Amount of capacity expansion $s$ in bio-refinery $j$ in period $t$

\section{Binary decision variables}

$\begin{array}{ll}x_{f}^{p} & \begin{array}{l}1 \text { if location } f \text { with capacity level } p \text { is selected for opening a JCL supply center; } 0 \\ \text { otherwise }\end{array} \\ y_{i t} & 1 \text { if location } i \text { is selected for opening an oil extraction center in period } t ; 0 \text { otherwise } \\ u_{j t} & 1 \text { if location } j \text { is selected for opening a bio-refinery in period } t \text {; } 0 \text { otherwise } \\ y e_{i t}^{q} & 1 \text { if oil extraction center } i \text { is undergone capacity expansion equal to } q \text { in period } t \text {; otherwise } \\ & 0\end{array}$


$u e_{j t}^{S} \quad 1$ if bio-refinery $j$ is undergone capacity expansion equal to $s$ in period $t ; 0$ otherwise

\section{Continuous decision variables}

$B O_{j t} \quad$ Amount of produced biodiesel at bio-refinery $j$ in period $t$ (ton/period)

$G L_{j t} \quad$ Amount of produced glycerin at bio-refinery $j$ in period $t$ (ton/period)

$J_{i t} \quad$ Amount of produced JCL oil at oil extraction center $i$ in period $t$ (ton/period)

$J a_{\text {flit }} \quad$ Transported amount of JCL yields from supply center $f$ to oil extraction center $i$ by mode $l$ in period $t$ (ton/period) $\begin{array}{ll}W_{\text {gljt }} & \text { Transported amount of WCO from WCO supply center } g \text { to bio-refinery } j \text { by mode } l \text { in } \\ \text { period } t \text { (ton/period) }\end{array}$

$O_{i l j t} \quad$ Transported amount of JCL oil from oil extraction center $i$ to bio-refinery $j$ by model $l$ in period $t$ (ton/period)

$B_{j l k t} \quad$ Transported amount of biodiesel from bio-refinery $j$ to consumer center $k$ by mode $l$ in period $t$ (ton/period)

$\mathrm{CO}_{i t} \quad$ Total capacity of oil extraction center $i$ in period $t$ (ton)

$C B_{j t} \quad$ Total capacity of bio-refinery $j$ in period $t$ (ton)

$I_{i t} \quad$ Amount of imported JCL seeds to oil extraction center $i$ in time period $t$ (ton)

In terms of the above notations, the MILP model for the given second-generation biodiesel supply chain network design problem is formulated as follows.

$$
\begin{aligned}
\operatorname{Min} Z= & \sum_{f, p} \widetilde{F J}_{f}^{p} \cdot x_{f}^{p}+\sum_{i, t} \widetilde{F O}_{i t} \cdot\left(y_{i t}-y_{i(t-1)}\right)+\sum_{j, t} \widetilde{F B}_{j t} \cdot\left(u_{j t}-u_{j(t-1)}\right) \\
& +\sum_{i, t, q} \widetilde{V O}_{i t}^{q} \cdot y e_{i t}^{q}+\sum_{j, t} \widetilde{V B}_{j t}^{s} \cdot u e_{j t}^{s}+\sum_{f, l, i, t} \widetilde{J T}_{f l i t} \cdot J a_{f l i t} \\
& +\sum_{i, l, j, t}\left(\widetilde{P O}_{i t}+\widetilde{O T}_{i l j t}\right) \cdot O_{i l j t}+\sum_{g, l, j, t} \widetilde{W T}_{g l j t} \cdot W_{g l j t} \\
& +\sum_{j, l, k, t}\left(\widetilde{P B}_{j t}+\widetilde{B T}_{j l k t}\right) \cdot B_{j l k t}+\sum_{i, t} \widetilde{P I}_{i t} \cdot I m_{i t}-\sum_{j, t} \widetilde{P G}_{j t} \cdot G L_{j t} \\
\sum_{j, l} B_{j l k t} & \geq \widetilde{D}_{k t} \forall k, t \\
\sum_{l, j} W_{g l j t} & =W \widetilde{O}_{g t} \forall g, t \\
\sum_{l, i} J a_{f l i t} & =\tilde{\eta}_{f t} \sum_{p} C J_{f}^{p} \cdot x_{f}^{p} \quad \forall f, t \\
\sum_{l, j} O_{i l j t} & =\varphi \times\left(\sum_{f, l} J a_{f l i t}+I m_{i t}\right) \quad \forall i, t
\end{aligned}
$$




$$
\begin{aligned}
& \sum_{l, k} B_{j l k t}=\beta \times\left(\sum_{g, l} W_{g l j t}+\sum_{i, l} O_{i l j t}\right) \quad \forall j, t \\
& G L_{j t}=(1-\beta) \times\left(\sum_{g, l} W_{g l j t}+\sum_{i, l} O_{i l j t}\right) \quad \forall j, t \\
& y_{i t} \leq y_{i(t+1)} \quad \forall i, t \\
& u_{j t} \leq u_{j(t+1)} \quad \forall j, t \\
& y e_{i t}^{q} \leq y_{i t} \quad \forall i, t, q \\
& u e_{j t}^{S} \leq u_{j t} \quad \forall j, t, s \\
& \sum_{q} y e_{i t}^{q} \leq 1 \quad \forall i, t \\
& \sum_{s} u e_{j t}^{s} \leq 1 \quad \forall j, t \\
& \sum_{p} x_{f}^{p} \leq 1 \quad \forall f \\
& \sum_{i, t}\left(y_{i t}-y_{i(t-1)}\right) \leq \operatorname{Maxy} \\
& \sum_{j, t}\left(u_{j t}-u_{j(t-1)}\right) \leq \operatorname{Maxu} \\
& I_{i t} \leq M I_{i t} \quad \forall i, t \\
& C O_{i t}=C O_{i(t-1)}+I C O_{i t} \cdot\left(y_{i t}-y_{i(t-1)}\right)+\sum_{q} y e_{i t}^{q} \cdot C E O_{i t}^{q} \\
& C B_{j t}=C B_{j(t-1)}+I C B_{j t} \cdot\left(u_{i t}-u_{i(t-1)}\right)+\sum_{s} u e_{j t}^{s} \cdot C E B_{j t}^{s} \quad \forall j, t \\
& \sum_{q, t} y e_{i t}^{q} \cdot C E O_{i t}^{q} \leq U O_{i} \quad \forall i \\
& \sum_{s, t} u e_{j t}^{s} \cdot C E B_{j t}^{S} \leq U B_{j} \quad \forall j \\
& \sum_{f, l} J a_{f l i t}+I m_{i t} \leq C O_{i t} \quad \forall i, t \\
& \sum_{g, l} W_{g l j t}+\sum_{i, l} O_{i l j t} \leq C B_{j t} \quad \forall j, t \\
& x_{f}^{p}, y_{i t}^{q}, u_{j t}^{s}, y e_{i t}^{q}, u e_{j t}^{s} \in\{0,1\} \\
& \text { Continuous decision variables } \geq 0
\end{aligned}
$$

Objective function (1) minimizes total costs of opening facilities, capacity expansions, production, transportation, and import and maximizes the income from selling glycerin. Constraint (2) satisfies biodiesel demand in all time periods. Constraints (3) and (4) ensure that all JCL feedstock and WCO are completely used for biodiesel production. Equation (5) shows the amount of produced JCL oil from JCL seeds that are supplied from internal supply centers and external suppliers. Equations (6) and (7) show the 
amount of produced biodiesel and glycerin from JCL oil or WCO, respectively. Constraints (8) and (9) ensure that the opened facilities remain active by the end of planning horizon. Constraints (10) and (11) demonstrate that capacity expansion variables depend on opening facility variables. Constraints (12) and (13) illustrate that at most, one capacity expansion level can be selected in the next periods. Constraint (14) ensures that only one of the capacity levels considered for established JCL supply centers can be selected. Constraints (15) and (16) bind the maximum number of facilities that can be established. This constraint is considered due to budget limitation. Constraint (17) binds the maximum amount of JCL that can be imported from external suppliers. This constraint is considered so as to preserve the independence of JCL supply from external suppliers in disasters. Constraints (18) and (19) show the capacity required for oil extraction centers and bio-refineries. Given the fact that JCL yields after two years of cultivation and in order to keep continuous yielding of JCL trees, it is assumed that the capacity of JCL cultivation areas does not depend on time periods. In contrast, the capacity of other facilities is dependent on time periods to be expanded according to the increase of feedstock resources. Constraints (20) and (21) consider the upper bound of capacity expansion of established facilities. Eqs. (22) and (23) demonstrate capacity constraint for oil extraction centers and bio-refineries, respectively. Eqs. (24) and (25) enforce non-negativity and binary constraints on the corresponding decision variables of the model.

\subsection{The proposed credibility-based fuzzy chance constrained programming approach}

Due to the epistemic uncertainty in the given problem, possibility distributions are applied in order to model the uncertain parameters. Therefore, possibilistic programming approaches should be used for dealing with these possibilistic data. Here, a credibility-based fuzzy chance constrained programming approach [37], one of the most confident possibilistic programming approaches in the literature, is used so as to handle the imprecise parameters in the proposed model. credibility-based fuzzy chance constrained programming is a credible possibilistic programming approach that relies on strong mathematical concepts such as the expected value of a possibilistic number and the credibility measure, and makes the decision maker (DM) able to control the confidence level of constraints' satisfactions in addition to supporting different types of possibilistic numbers such as triangular and trapezoidal forms [38]. It is worth mentioning that in contrast to the possibility and necessity measures that do not have self-duality property, the credibility measure is a self-dual measure [37]. That is, if the credibility value of a fuzzy event is 1, DM believes that the fuzzy event will definitely happen; nevertheless, when the corresponding possibility measure is 1 , the fuzzy event may fail to happen and when its necessity measure equals to 0, the fuzzy event may happen [38].

Suppose that $\tilde{\xi}$ is a fuzzy variable with membership function $\mu(x)$, and let $r$ be a real number. According to Liu and Liu [39], the credibility measure is defined as follows:

$$
C r\{\tilde{\xi} \leq r\}=\frac{1}{2}\left(\sup _{x \leq r} \mu(x)+1-\sup _{x>r} \mu(x)\right)
$$

Since $\operatorname{Pos}\{\tilde{\xi} \leq r\}=\sup _{x \leq r} \mu(x)$ and $\operatorname{Nec}\{\tilde{\xi} \leq r\}=1-\sup _{x>r} \mu(x)$, credibility measure can be defined as follows: 


$$
\operatorname{Cr}\{\tilde{\xi} \leq r\}=\frac{1}{2}(\operatorname{Pos}\{\tilde{\xi} \leq r\}+\operatorname{Nec}\{\tilde{\xi} \leq r\})
$$

Also, according to the definition of credibility measure, the expected value of $\tilde{\xi}$ can be determined as follows:

$$
E[\tilde{\xi}]=\int_{0}^{\infty} c r\{\tilde{\xi} \geq r\} d r-\int_{-\infty}^{0} c r\{\tilde{\xi} \leq r\} d r
$$

Now, suppose $\tilde{\xi}=\left(\xi^{(1)}, \xi^{(2)}, \xi^{(3)}, \xi^{(4)}\right)$ is trapezoidal fuzzy number. According to the Eq. (28), the expected value of $\tilde{\xi}$ is calculated as follows:

$$
E[\tilde{\xi}]=\frac{\xi^{(1)}+\xi^{(2)}+\xi^{(3)}+\xi^{(4)}}{4}
$$

Also, the corresponding credibility measures of $\tilde{\xi}$ are as follows:

$$
\begin{aligned}
& \operatorname{cr}\{\tilde{\xi} \leq r\}= \begin{cases}0 & r \in\left(-\infty, \xi^{(1)}\right], \\
\frac{r-\xi^{(1)}}{2\left(\xi^{(2)}-\xi^{(1)}\right)} & r \in\left(\xi^{(1)}, \xi^{(2)}\right], \\
\frac{1}{2} & r \in\left(\xi^{(2)}, \xi^{(3)}\right], \\
\frac{r-2 \xi^{(3)}+\xi^{(4)}}{2\left(\xi^{(4)}-\xi^{(3)}\right)} & r \in\left(\xi^{(3)}, \xi^{(4)}\right], \\
1 & r \in\left(\xi^{(4)},+\infty\right) .\end{cases} \\
& \operatorname{cr}\{\tilde{\xi} \geq r\}= \begin{cases}1 & r \in\left(-\infty, \xi^{(1)}\right], \\
\frac{2 \xi^{(2)}-\xi^{(1)}-r}{2\left(\xi^{(2)}-\xi^{(1)}\right)} & r \in\left(\xi^{(1)}, \xi^{(2)}\right], \\
\frac{1}{2} & r \in\left(\xi^{(2)}, \xi^{(3)}\right], \\
\frac{\xi^{(4)}-r}{2\left(\xi^{(4)}-\xi^{(3)}\right)} & r \in\left(\xi^{(3)}, \xi^{(4)}\right], \\
0 & r \in\left(\xi^{(4)},+\infty\right) .\end{cases}
\end{aligned}
$$

Thus, based on Eqs. (30) and (31), if $\alpha>0.5$ then [40]:

$$
\begin{aligned}
& \operatorname{cr}\{\tilde{\xi} \leq r\} \geq \alpha \Leftrightarrow r \geq(2-2 \alpha) \xi^{(3)}+(2 \alpha-1) \xi^{(4)} \\
& \operatorname{cr}\{\tilde{\xi} \geq r\} \geq \alpha \Leftrightarrow r \leq(2 \alpha-1) \xi^{(1)}+(2-2 \alpha) \xi^{(2)} .
\end{aligned}
$$


Eqs. (32) and (33) can be used for converting the fuzzy inequality chance constraints to their equivalents crisp ones.

Based on Eqs. (32) and (33), if $\xi^{(2)} \leq r \leq \xi^{(3)}$ then:

$$
\begin{aligned}
& \operatorname{cr}\{\tilde{\xi} \leq r\}=\frac{1}{2} \\
& \operatorname{cr}\{\tilde{\xi} \geq r\}=\frac{1}{2}
\end{aligned}
$$

It can be concluded from Eqs. (34) and (35) that $r$ and $\tilde{\xi}$ are indifferent or approximately equal [41].

Definition 1. The real number $r$ and trapezoidal fuzzy number $\tilde{\xi}$ are indifferent or approximately equal if and only if

$$
\tilde{\xi} \approx r \quad \Leftrightarrow \quad \xi^{(2)} \leq r \leq \xi^{(3)}
$$

In the literature, there are three kinds of credibility-based fuzzy mathematical programming models, including (1) expected value [39], (2) chance constrained programming [42] and (3) dependent-chance constrained programming [43]. The expected value model is the simplest one that does not increase the computational complexity of the original model; however, it does not control the confidence level of chance constraints' satisfactions. By contrast, the chance constrained programming models can control the satisfaction degree of chance constraints; nonetheless, it increases the computational complexity of the original model because it adds a constraint for each objective function and requires additional information or experts' judges about the ideal value of objective functions in order to determine the right hand side of the added constraints. The dependent-chance programming model that is similar to chance constrained programming model is more suitable for decision making in conservative situations, inasmuch as it gives more importance to confidence levels. In this paper, the approach proposed by [41] is used for dealing with uncertain parameters. In this approach, the expected value and the chance constrained programming models are combined in order to form a credibility-based fuzzy mathematical programming framework for the given problem. The expected value is applied to model the objective functions and the chance constrained programming approach is used for modeling the chance constraints including uncertain parameters. This approach not only has the advantages of the three other approaches, but it also does not increase the complexity of the model. This approach does not need additional information about the ideal value of the objective function. In addition, it does not increase the number of the constraints of the initial model and its complexity. Additionally, in this approach, the satisfaction degree of constraints can be controlled by virtue of the advantages of chance constrained programming.

According to the above-mentioned explanations, the proposed credibility-based possibilistic programming model for the given second-generation biodiesel supply chain network design problem is presented as follows.

$$
\operatorname{Min} Z 1=\sum_{f, p} E\left[\widetilde{F J}_{f}^{p}\right] \cdot x_{f}^{p}+\sum_{i, t} E\left[\widetilde{F O}_{i t}\right] \cdot\left(y_{i t}-y_{i(t-1)}\right)+\sum_{j, t} E\left[\widetilde{F B}_{j t}\right] \cdot\left(u_{j t}-u_{j(t-1)}\right)
$$




$$
\begin{aligned}
& +\sum_{i, t, q} E\left[\widetilde{V O}_{i t}^{q}\right] \cdot y e_{i t}^{q}+\sum_{j, t} E\left[\widetilde{V B}_{j t}^{s}\right] \cdot u e_{j t}^{s}+\sum_{f, l, i, t} E\left[\widetilde{J T}_{f l i t}\right] \cdot J a_{f l i t} \\
& +\sum_{i, l, j, t} E\left[\left(\widetilde{P O}_{i t}+\widetilde{O T}_{i l j t}\right)\right] \cdot O_{i l j t}+\sum_{g, l, j, t} E[\widetilde{W T} \widetilde{g l j t}] \cdot W_{g l j t} \\
& +\sum_{j, l, k, t} E\left[\left(\widetilde{P B}_{j t}+\widetilde{B T}_{j l k t}\right)\right] \cdot B_{j l k t}+\sum_{i, t} E\left[\widetilde{P I}_{i t}\right] \cdot I m_{i t}-\sum_{j, t} E\left[\widetilde{P G}_{j t}\right] \cdot G L_{j t} \\
& \operatorname{Cr}\left\{\sum_{j, l} B_{j l k t} \geq \widetilde{D}_{k t}\right\} \geq \alpha_{k t}, \quad \forall k, t \\
& \operatorname{Cr}\left\{\sum_{l, j} W_{g l j t} \geq W \tilde{O}_{g t}\right\}=\frac{1}{2}, \quad \forall g, t \\
& \operatorname{Cr}\left\{\sum_{l, j} W_{g l j t} \leq W \tilde{O}_{g t}\right\}=\frac{1}{2}, \quad \forall g, t \\
& \operatorname{Cr}\left\{\sum_{l, i} J a_{f l i t} \geq \tilde{\eta}_{f t} \sum_{p} C J_{f}^{p} \cdot x_{f}^{p}\right\}=\frac{1}{2} \quad \forall f, t \\
& \operatorname{Cr}\left\{\sum_{l, i} J a_{f l i t} \leq \tilde{\eta}_{f t} \sum_{p} C J_{f}^{p} \cdot x_{f}^{p}\right\}=\frac{1}{2} \quad \forall f, t \\
& \sum_{l, j} O_{i l j t}=\varphi \times\left(\sum_{f, l} J a_{f l i t}+I m_{i t}\right) \quad \forall i, t \\
& \sum_{l, k} B_{j l k t}=\beta \times\left(\sum_{g, l} W_{g l j t}+\sum_{i, l} O_{i l j t}\right) \quad \forall j, t \\
& G L_{j t}=(1-\beta) \times\left(\sum_{g, l} W_{g l j t}+\sum_{i, l} O_{i l j t}\right) \quad \forall j, t \\
& y_{i t} \leq y_{i(t+1)} \quad \forall i, t \\
& u_{j t} \leq u_{j(t+1)} \quad \forall j, t \\
& y e_{i t}^{q} \leq y_{i t} \quad \forall i, t, q \\
& u e_{j t}^{s} \leq u_{j t} \quad \forall j, t, s \\
& \sum_{q} y e_{i t}^{q} \leq 1 \quad \forall i, t \\
& \sum_{s} u e_{j t}^{s} \leq 1 \quad \forall j, t \\
& \sum_{p} x_{f}^{p} \leq 1 \quad \forall f \\
& \sum_{i, t}\left(y_{i t}-y_{i(t-1)}\right) \leq \operatorname{Max} y
\end{aligned}
$$




$$
\begin{aligned}
& \sum_{j, t}\left(u_{j t}-u_{j(t-1)}\right) \leq \operatorname{Maxu} \\
& I m_{i t} \leq M I_{i t} \quad \forall i, t \\
& C O_{i t}=C O_{i(t-1)}+I C O_{i t} \cdot\left(y_{i t}-y_{i(t-1)}\right)+\sum_{q} y e_{i t}^{q} \cdot C E O_{i t}^{q} \quad \forall i, t \\
& C B_{j t}=C B_{j(t-1)}+I C B_{j t} \cdot\left(u_{i t}-u_{i(t-1)}\right)+\sum_{s} u e_{j t}^{s} . C E B_{j t}^{s} \quad \forall j, t \\
& \sum_{q, t} y e_{i t}^{q} \cdot C E O_{i t}^{q} \leq U O_{i} \quad \forall i \quad \forall j \quad \forall i, t \\
& \sum_{s, t} u e_{j t}^{s} \cdot C E B_{j t}^{s} \leq U B_{j} \quad \forall j, t \\
& \sum_{f, l}^{J} a_{f l i t}+I m_{i t} \leq C O_{i t} \quad \forall \\
& \sum_{g, l} W_{g l j t}+\sum_{i, l} O_{i l j t} \leq C B_{j t} \quad \forall \\
& x_{f}^{p}, y_{i t}^{q}, u_{j t}^{s}, y e_{i t}^{q}, u e_{j t}^{s} \in\{0,1\} \\
& \text { Continuous decision variables } \geq 0
\end{aligned}
$$

The above model can be transformed to the equivalent crisp one through the presented definitions and with the assumption $\alpha>0.5$ for chance constraints. The final model is an MILP model that is presented as follows.

$$
\begin{aligned}
\text { Min } Z 1 & =\sum_{f, p}\left[\frac{F J_{f}^{p(1)}+F J_{f}^{p(2)}+F J_{f}^{p(3)}+F J_{f}^{p(4)}}{4}\right] \cdot x_{f}^{p} \\
& +\sum_{i, t}\left[\frac{F O_{i t}^{(1)}+F O_{i t}^{(2)}+F O_{i t}^{(3)}+F O_{i t}^{(4)}}{4}\right] \cdot\left(y_{i t}-y_{i(t-1)}\right) \\
& +\sum_{j, t}\left[\frac{F B_{j t}^{(1)}+F B_{j t}^{(2)}+F B_{j t}^{(3)}+F B_{j t}^{(4)}}{4}\right] \cdot\left(u_{j t}-u_{j(t-1)}\right) \\
& +\sum_{i, t, q}\left[\frac{V O_{i t}^{q(1)}+V O_{i t}^{q(2)}+V O_{i t}^{q(3)}+V O_{i t}^{q(4)}}{4}\right] \cdot y e_{i t}^{q} \\
& +\sum_{j, t}\left[\frac{V B_{j t}^{s(1)}+V B_{j t}^{s(2)}+V B_{j t}^{s(3)}+V B_{j t}^{s(4)}}{4}\right] \cdot u e_{j t}^{s} \\
& +\sum_{f, l, i, t}\left[\frac{J T_{f l i t}^{(1)}+J T_{f l i t}^{(2)}+J T_{f l i t}^{(3)}+J T_{f l i t}^{(4)}}{4}\right] \cdot J a_{f l i t} \\
& +\sum_{i, l, j, t}\left[\frac{P O_{i t}^{(1)}+P O_{i t}^{(2)}+P O_{i t}^{(3)}+P O_{i t}^{(4)}}{4}+\frac{O T_{i l j t}^{(1)}+O T_{i l j t}^{(2)}+O T_{i l j t}^{(3)}+O T_{i l j t}^{(4)}}{4}\right] . O_{i l j t}
\end{aligned}
$$




$$
\begin{aligned}
& +\sum_{g, l, j, t}\left[\frac{W T_{g l j t}^{(1)}+W T_{g l j t}^{(2)}+W T_{g l j t}^{(3)}+W T_{g l j t}^{(4)}}{4}\right] \cdot W_{g l j t} \\
& +\sum_{j, l, k, t}\left[\frac{P B_{j t}^{(1)}+P B_{j t}^{(2)}+P B_{j t}^{(3)}+P B_{j t}^{(4)}}{4}+\frac{B T_{j l k t}^{(1)}+B T_{j l k t}^{(2)}+B T_{j l k t}^{(3)}+B T_{j l k t}^{(4)}}{4}\right] \cdot B_{j l k t} \\
& +\sum_{i, t}\left[\frac{P I_{i t}^{(1)}+P I_{i t}^{(2)}+P I_{i t}^{(3)}+P I_{i t}^{(4)}}{4}\right] \cdot I m_{i t} \\
& -\sum_{j, t}\left[\frac{P G_{j t}^{(1)}+P G_{j t}^{(2)}+P G_{j t}^{(3)}+P G_{j t}^{(4)}}{4}\right] \cdot G L_{j t} \\
& \sum_{j, l} B_{j l k t} \geq\left(2-2 \alpha_{k t}\right) D_{k t}^{(3)}+\left(2 \alpha_{k t}-1\right) D_{k t}^{(4)}, \quad \forall k, t \\
& W O_{g t}^{(2)} \leq \sum_{l, j} W_{g l j t} \quad \forall g, t \\
& \sum_{l, j} W_{g l j t} \leq W O_{g t}^{(3)} \quad \forall g, t \\
& \eta_{f t}^{(2)} \sum_{p} C J_{f}^{p} \cdot x_{f}^{p} \leq \sum_{l, i} J a_{f l i t} \quad \forall f, t \\
& \sum_{l, i} J a_{f l i t} \leq \eta_{f t}^{(3)} \sum_{p} C J_{f}^{p} \cdot x_{f}^{p} \quad \forall f, t \\
& \sum_{l, j} O_{i l j t}=\varphi \times\left(\sum_{f, l} J a_{f l i t}+I m_{i t}\right) \quad \forall i, t \\
& \sum_{l, k} B_{j l k t}=\beta \times\left(\sum_{g, l} W_{g l j t}+\sum_{i, l} O_{i l j t}\right) \quad \forall j, t \\
& G L_{j t}=(1-\beta) \times\left(\sum_{g, l} W_{g l j t}+\sum_{i, l} O_{i l j t}\right) \quad \forall j, t \\
& y_{i t} \leq y_{i(t+1)} \quad \forall i, t \\
& u_{j t} \leq u_{j(t+1)} \quad \forall j, t \\
& y e_{i t}^{q} \leq y_{i t} \quad \forall i, t, q \\
& u e_{j t}^{s} \leq u_{j t} \quad \forall j, t, s \\
& \sum_{q} y e_{i t}^{q} \leq 1 \quad \forall i, t \\
& \sum_{s} u e_{j t}^{s} \leq 1 \quad \forall j, t \\
& \sum_{p} x_{f}^{p} \leq 1 \quad \forall f
\end{aligned}
$$




$$
\begin{aligned}
& \sum_{i, t}\left(y_{i t}-y_{i(t-1)}\right) \leq \operatorname{Maxy} \\
& \sum_{j, t}\left(u_{j t}-u_{j(t-1)}\right) \leq \operatorname{Maxu} \\
& I_{i t} \leq M I_{i t} \quad \forall i, t \\
& C O_{i t}=C O_{i(t-1)}+I C O_{i t} \cdot\left(y_{i t}-y_{i(t-1)}\right)+\sum_{q} y e_{i t}^{q} \cdot C E O_{i t}^{q} \\
& C B_{j t}=C B_{j(t-1)}+I C B_{j t} \cdot\left(u_{i t}-u_{i(t-1)}\right)+\sum_{s} u e_{j t}^{S} \cdot C E B_{j t}^{S} \quad \forall j, t \\
& \sum_{q, t} y e_{i t}^{q} \cdot C E O_{i t}^{q} \leq U O_{i} \quad \forall i \\
& \sum_{s, t} u e_{j t}^{s} \cdot C E B_{j t}^{S} \leq U B_{j} \quad \forall j \\
& \sum_{f, l} J a_{f l i t}+I m_{i t} \leq C O_{i t} \quad \forall i, t \\
& \sum_{g, l} W_{g l j t}+\sum_{i, l} o_{i l j t} \leq C B_{j t} \quad \forall j, t \\
& x_{f}^{p}, y_{i t}^{q}, u_{j t}^{s}, y e_{i t}^{q}, u e_{j t}^{s} \in\{0,1\} \\
& \text { Continuous decision variables } \geq 0
\end{aligned}
$$

\section{Solution approach}

\subsection{Benders decomposition algorithm}

According to the Davis and Ray [44] and Mirchandani and Francis [45], facility location problem is an NPhard problem. Accordingly, our proposed model that is an extension of a facility location problem is NPhard. In order to decrease the computational complexity of the proposed model, in this section, an Accelerated Benders Decomposition Algorithm (ABDA) is developed. BDA, introduced by Benders [46], is an efficient algorithm to solve large-scale MIP problems. In BDA, we decompose the original complex MIP problem into a pure integer programming problem (master problem) and a linear programming problem (sub-problem). The master problem (MP) and sub-problem (SP) are solved iteratively through using the optimum solution of one problem in the other. In order to develop a BDA for the given model, first we should formulate the dual sub-problem (DSP) and MP. Due to the fact that integer programming models do not have a dual form, in order to formulate the dual form of these models, integer variables should be freed. MS and DSP provide upper bound and lower bound of the optimal solution and converge on the optimal solution under an iterative procedure. The description of BDA is presented as follows.

Consider the following MILP model in which Y indicates the set of integer numbers. The following is known as the main model.

$$
\text { Min } c^{T} x+f^{T} y
$$


S.t. $\quad A x+B y \geq b$

$$
\begin{aligned}
& y \in Y \\
& x \geq 0
\end{aligned}
$$

In the above model, if integer variable $y$ is fixed to a feasible value, we will have the following linear model:

Min $c^{T} x$

S.t. $\quad A x \geq b-B y$

$$
x \geq 0
$$

Therefore, for all feasible values of $y$, Primal Sub Problem (PSP) is formulated as follows:

$$
\operatorname{Min}_{y \in Y}\left[f^{T} y+\min _{x \geq 0}\left\{c^{T} x \mid A x \geq b-B y\right\}\right]
$$

Due to the fact that MILP models do not have the dual form, through fixing the integer variable $y$, the dual form of the above linear model is presented as follows. $u$ is the dual variable related to the constraint of the PSP.

$$
\operatorname{Max}_{u}(b-B \bar{y})^{T} u
$$

S.t. $\quad A^{T} u \leq c$

$$
u \geq 0
$$

The above linear model is known as the DSP. Since the optimal solution of the PSP and DSP are the same, the PSP is equivalent to the following problem.

$$
\operatorname{Min}_{y \in Y}\left[f^{T} y+\max _{u \geq 0}\left\{(b-B \bar{y})^{T} u \mid A^{T} u \leq c\right\}\right]
$$

The solution space of the above problem is independent from integer variable $y$. If the PSP has feasible solution, the solution space of the DSP will not be empty. If the PSP is infeasible or unbounded, the solution space of the DSP will be empty. The PSP's being infeasible or unbounded shows the wrong modeling of this problem. Accordingly, if the problem is modeled in an accurate way, the DSP will have bounded or unbounded solution space. That is, generally, the solution space of the DSP has extreme points $\left(u^{p}\right)$ and extreme rays $\left(r^{q}\right)$. If it has a bounded solution space, the solution will be one of the extreme points $\left(u^{p}\right)$ and if it has an unbounded solution space, there is an extreme ray $\left(r^{q}\right)$ for which $r^{q}(b-B \bar{y})^{T}>0$. Inasmuch as the DSP's being unbounded results in the infeasibility of the PSP, for preventing the DSP from becoming unbounded, the following constraint is added to this problem.

$$
r^{q}(b-B \bar{y})^{T} \leq 0 \quad \forall q
$$


Based on the above explanations, using the dummy variable $z$, the PSP is reformulated as follows.

Min $z+f^{T} y$

S.t. $\quad z \geq(b-B y)^{T} \bar{u}^{p}, \quad \forall p$

$$
\begin{aligned}
& r^{q}(b-B y)^{T} \leq 0 \quad \forall q \\
& y \in Y \\
& z \geq 0
\end{aligned}
$$

The above problem is known as the main problem. In this problem, $u$ and $r$ are the parameters, the value of which can be determined by the dual model; then, they are used in the main problem. The main problem includes only integer variables and is an integer programming problem.

In the BDA, first we find a feasible integer value $(\bar{y})$ for the variable; then, use it in the DSP and solve the DSP. If the obtained solution is bounded, we formulate the main problem and add the constraint $z \geq(b-B y)^{T} \bar{u}^{p}$, known as optimality cut, to the main problem. After solving the main problem, if the solution is unbounded, a constraint as $r^{q}(b-B y)^{T} \leq 0$, known as feasibility cut, is added to the main problem; afterwards, the model is solved again. This iterative procedure continues until the algorithm converges on an optimal value. In a minimization problem, in each iteration of the BDA, DSP and MP produce an upper bound and lower bound for the original problem, respectively. The iteration of the algorithm continues so much so that the upper and lower bounds become the same, which means that we have reached to the optimal solution. According to the above descriptions, the procedure of the BDA for solving MILP is as follows.

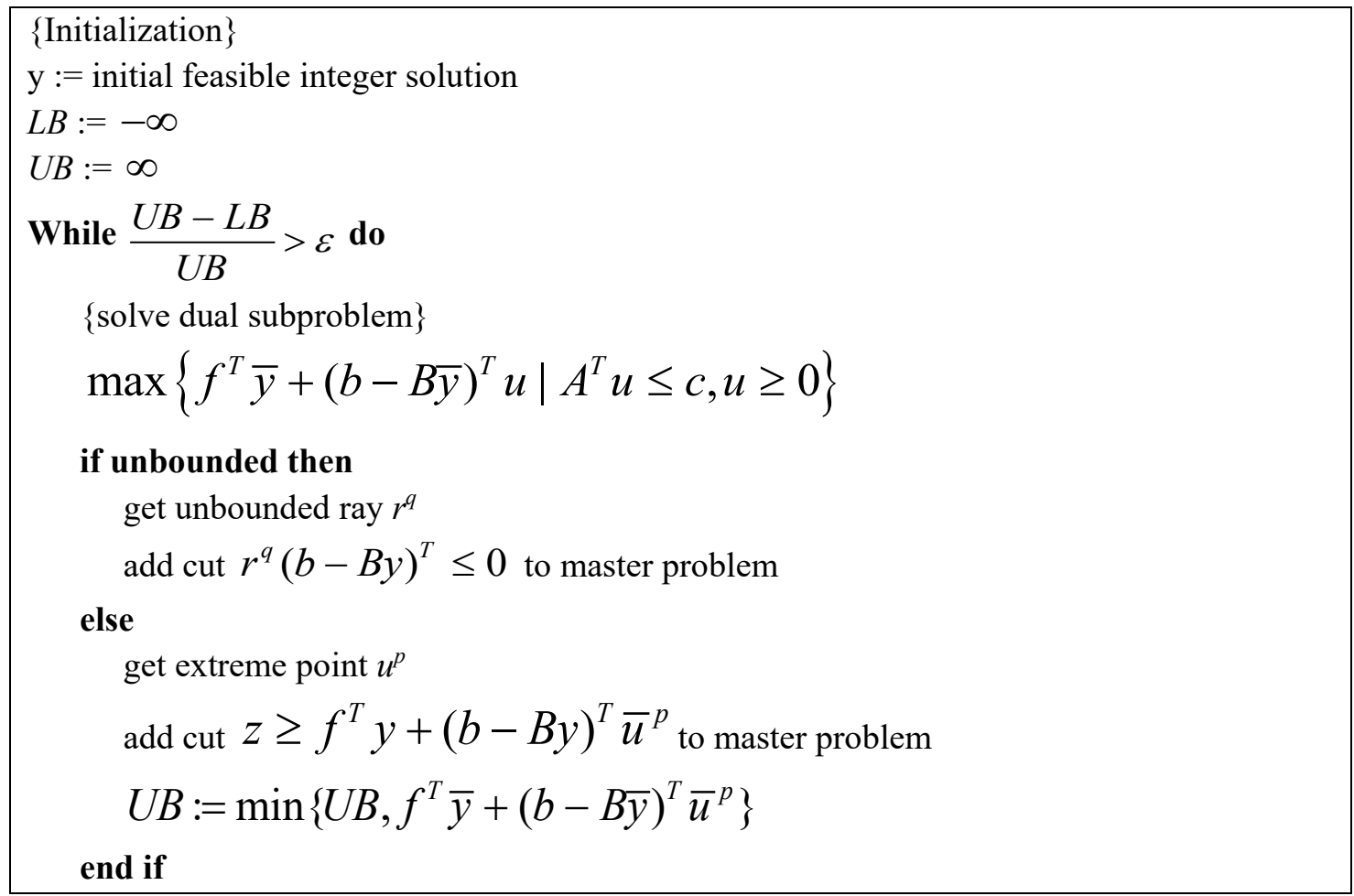




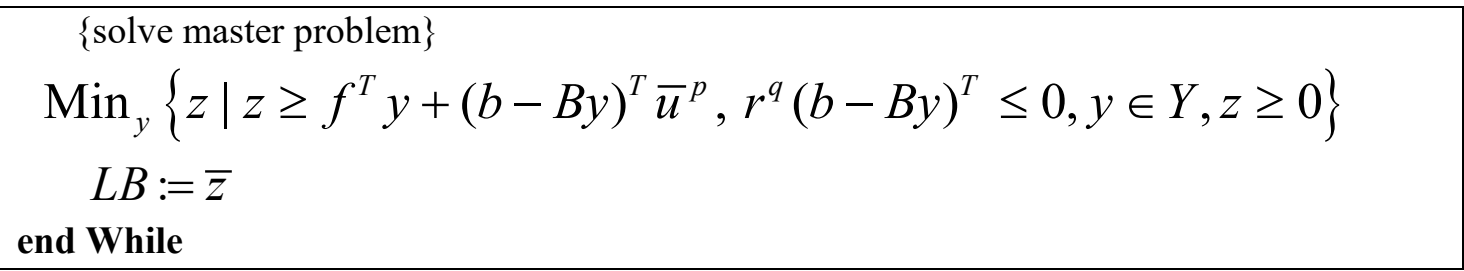

It is worthy to note that the classical BDA usually fails to provide strong lower bound and upper bound for the main problem and converges in many iterations and large computational time. To deal with this deficiency of BDA, some researchers have added efficient and strong cuts to MP and solve the main problems in low number of iterations and efficient computational time. This method is well known to ABDA. In this paper, local branching cuts are added to MP to accelerate the BDA. Also, an integer programming model equipped with valid inequalities is proposed to find efficient feasible solution to run the ABDA. According to above description, the MP and DSP for the proposed possibilistic programming model could be formed according to Appendix A.

\subsection{Finding the initial feasible solution}

The first step of BDA is finding an initial feasible solution. Usually, in order to begin the BDA, an initial solution equal to 0 or 1 is considered for binary variables in the MILP problems, and in doing so, the number of iterations increases. Therefore, finding a high quality initial solution can significantly decrease the solution time of BDA. In the given model, the feasible solution of integer variables is obtained through solving the following model. The following model is a pure integer programming model, including the constraints of the original problem except for optimality cut and valid constraints ensuring the feasibility of the solution.

$$
\begin{aligned}
\operatorname{Min} Z & =\sum_{f, p}\left[\frac{F J_{f}^{p(1)}+F J_{f}^{p(2)}+F J_{f}^{p(3)}+F J_{f}^{p(4)}}{4}\right] \cdot x_{f}^{p} \\
& +\sum_{i, t}\left[\frac{F O_{i t}^{(1)}+F O_{i t}^{(2)}+F O_{i t}^{(3)}+F O_{i t}^{(4)}}{4}\right] \cdot\left(y_{i t}-y_{i(t-1)}\right) \\
& +\sum_{j, t}\left[\frac{F B_{j t}^{(1)}+F B_{j t}^{(2)}+F B_{j t}^{(3)}+F B_{j t}^{(4)}}{4}\right] \cdot\left(u_{j t}-u_{j(t-1)}\right) \\
& +\sum_{i, t, q}\left[\frac{V O_{i t}^{q(1)}+V O_{i t}^{q(2)}+V O_{i t}^{q(3)}+V O_{i t}^{q(4)}}{4}\right] \cdot y e_{i t}^{q} \\
& +\sum_{j, t}\left[\frac{V B_{j t}^{s(1)}+V B_{j t}^{s(2)}+V B_{j t}^{s(3)}+V B_{j t}^{s(4)}}{4}\right] \cdot u e_{j t}^{s}
\end{aligned}
$$

s.t.

$\beta . \sum_{j} C B_{j t} \geq \sum_{k}\left(\left(2-2 \alpha_{k t}\right) D_{k t}^{(3)}+\left(2 \alpha_{k t}-1\right) D_{k t}^{(4)}\right) \quad \forall t$

$\beta \cdot\left(\varphi \cdot \sum_{i} C O_{i t}+\sum_{g} W O_{g t}^{(3)}\right) \geq \sum_{k}\left(\left(2-2 \alpha_{k t}\right) D_{k t}^{(3)}+\left(2 \alpha_{k t}-1\right) D_{k t}^{(4)}\right) \quad \forall t$ 


$$
\begin{aligned}
& \beta . \varphi \cdot\left(\sum_{f, p} \eta_{f t}^{(3)} C J_{f}^{p} \cdot x_{f}^{p}+\sum_{i} M I_{i t}\right)+\beta \cdot \sum_{g} W O_{g t}^{(3)} \\
& \geq \sum_{k}\left(\left(2-2 \alpha_{k t}\right) D_{k t}^{(3)}+\left(2 \alpha_{k t}-1\right) D_{k t}^{(4)}\right) \quad \forall t \\
& y_{i t} \leq y_{i(t+1)} \quad \forall i, t \\
& u_{j t} \leq u_{j(t+1)} \quad \forall j, t \\
& y e_{i t}^{q} \leq y_{i t} \quad \forall i, t, q \\
& u e_{j t}^{s} \leq u_{j t} \quad \forall j, t, s \\
& \sum_{q} y e_{i t}^{q} \leq 1 \quad \forall i, t \\
& \sum_{s} u e_{j t}^{s} \leq 1 \quad \forall j, t \\
& \sum_{p}^{s} x_{f}^{p} \leq 1 \quad \forall f \\
& \sum_{i, t}\left(y_{i t}-y_{i(t-1)}\right) \leq \operatorname{Maxy} \\
& \sum_{j, t}\left(u_{j t}-u_{j(t-1)}\right) \leq \operatorname{Maxu} \\
& \sum_{q, t} y e_{i t}^{q} \cdot C E O_{i t}^{q} \leq U O_{i} \quad \forall i \\
& \sum_{s, t} u e_{j t}^{s} \cdot C E B_{j t}^{S} \leq U B_{j} \quad \forall j \\
& C O_{i t}=C O_{i(t-1)}+I C O_{i t} \cdot\left(y_{i t}-y_{i(t-1)}\right)+\sum_{q} y e_{i t}^{q} \cdot C E O_{i t}^{q} \quad \forall i, t \\
& C B_{j t}=C B_{j(t-1)}+I C B_{j t} \cdot\left(u_{j t}-u_{j(t-1)}\right)+\sum_{s} u e_{j t}^{s} \cdot C E B_{j t}^{s} \quad \forall j, t \\
& x_{f}^{p}, y_{i t}^{q}, u_{j t}^{s}, y e_{i t}^{q}, u e_{j t}^{s} \in\{0,1\}
\end{aligned}
$$

The feasible solution produced for the integer variables of the PSP are considered as the input parameters in the DSP; afterwards, the produced optimality cut is formed and MP is solved. Later, the solution of MP is used in the DSP. This procedure continues so that the lower and upper bounds converge on the optimal solution.

\subsection{Local branching}

The slow convergence of BDA is mainly because of the computational complexity of MP. DSP, a linear programming model, can be solved instantly. Nevertheless, MP is an integer programming model and adding new optimality or feasibility cuts in each iteration increases its complexity. Accordingly, solving MP needs considerable time. To address this issue, Geoffrion and Graves [47] demonstrated that it is not required to obtain the optimal solution of MP at each iteration. Rather, obtaining a near optimal solution could be beneficial. In order to use this idea, they developed a modified model for MP. Using this idea, Rei et al. [48] used a local branching method to efficiently solve complex MP in BDA. Local branching method was previously proposed by Fischetti and Lodi [49] so as to cope with complex integer programming 
models. In this method, the feasible space of MP is restricted through finding a reasonable neighborhood around the previous solution and then solving the restricted MP in order to obtain an optimal or a good feasible solution. By doing so, MP could be solved efficiently and considerable time would be saved.

Assume that $y^{r}$ is the solution (binary variable) obtained from MP at iteration $r$. The following constraint can be added to MP for restricting the feasible space in the next iteration.

$$
\Delta\left(y^{r}, y^{r+1}\right) \leq \gamma
$$

$\Delta$ indicates a suitable distance function and $\gamma$ denotes a positive number that is usually integer and takes a reasonable value. Adding this constraint to MP restricts the distance between the obtained solution at iteration $r+1$ and the obtained solution at iteration $r$ as much as $\gamma$. In this paper, in order to employ local branching method in the proposed BDA, Hamming [50] distance function is used. Also, branching is performed on the variables of establishing facilities.

Consider the following sets that show the values of the binary variables with value 1 .

$$
\begin{aligned}
& X^{r}:=\left\{f, p: x_{f}^{p}=1\right\} \\
& Y^{r}:=\left\{q, i, t:\left(y_{i t}^{q}-y_{i(t-1)}^{q}\right)=1\right\} \\
& W^{r}:=\left\{s, j, t:\left(u_{j t}^{s}-u_{j(t-1)}^{s}\right)=1\right\}
\end{aligned}
$$

Afterwards, the following constraint is added to the MP model.

$$
\begin{aligned}
& \sum_{f, p \in X^{r}}\left(1-x_{f}^{p}\right)+\sum_{f, p \notin X^{r}} x_{f}^{p}+\sum_{q, i, t \in Y^{r}}\left(1-\left(y_{i t}^{q}-y_{i(t-1)}^{q}\right)\right)+\sum_{q, i, t \notin Y^{r}}\left(y_{i t}^{q}-y_{i(t-1)}^{q}\right) \\
& \sum_{s, j, t \in W^{r}}\left(1-\left(u_{j t}^{s}-u_{j(t-1)}^{s}\right)\right)+\sum_{s, j, t \notin W^{r}}\left(u_{j t}^{s}-u_{j(t-1)}^{s}\right) \leq \gamma
\end{aligned}
$$

where $1 \leq \gamma \leq|F|+|I|+|J|$. It is worth mentioning that determining very small values for $\gamma$ causes infeasibility of MP. According to the above descriptions, the procedure of the ABDA for solving MILP is as follows.

$$
\begin{aligned}
& \{\text { Initialization }\} \\
& \mathrm{y}:=\text { initial feasible integer solution through applying the proposed pure IP model } \\
& L B:=-\infty \\
& U B:=\infty \\
& \text { While } \frac{U B-L B}{U B}>\varepsilon \text { do } \\
& \quad \text { solve dual subproblem }\} \\
& \quad \max \left\{f^{T} \bar{y}+(b-B \bar{y})^{T} u \mid A^{T} u \leq c, u \geq 0\right\} \\
& \quad \text { get extreme point } u^{p} \\
& \quad \text { add cut } z \geq f^{T} y+(b-B y)^{T} \bar{u}^{p} \text { to master problem }
\end{aligned}
$$




$\quad U B:=\min \left\{U B, f^{T} \bar{y}+(b-B \bar{y})^{T} \bar{u}^{p}\right\}$
end if
$\{$ solve master problem $\}$
$\operatorname{Min}_{y}\left\{z \mid z \geq f^{T} y+(b-B y)^{T} \bar{u}^{p}, y \in Y, z \geq 0\right\}$
add $\Delta\left(y^{r}, y^{r+1}\right) \leq \gamma$
$L B:=\bar{z}$
end While

\section{Case study}

In this section, the proposed credibility-based possibilistic programming model for the design of a secondgeneration biodiesel supply chain is implemented in real case study in Iran so that the performance and efficiency of the proposed model is evaluated.

Iran enjoys a great potential for producing quite a few biomass resources and energy crops such as sunflower, sorghum, sugar beet, switchgrass, and JCL. In fact, there are suitable climate conditions as well as waste lands in Iran which make it an appropriate place for agricultural activities. Additionally, Iran has a great amount of arid and semi-arid lands which could be used for cultivating energy crops such as JCL efficiently growing in marginal and infertile areas. According to the Jatropha belt $\left(30^{\circ} N, 35^{\circ} S\right.$ ), southern half of Iran is the best place for the cultivation of JCL. Iran imports about 80 percent of its edible oil consumption, which shows that using such edible oil resources as palm, sunflower, cotton, and soya for the production of biodiesel is irrational and would be to the detriment of food supply resources. Accordingly, the exploitation of other non-edible oil resources such as JCL oil and WCO for biodiesel production is an excellent opportunity for the development of biodiesel production in Iran. WCO is another non-edible oil that can be used as a great resource for biodiesel production in Iran. It is worth mentioning that the annual production rate of WCO in Iran is 300,000 tons. In Iran, there is currently one bio-refinery for biodiesel production in Isfahan city. Iran is planning to produce different kinds of renewable energies and decrease dependency on crude oil. The evaluation of renewable energy consumption in Iran shows that it has had a growing trend in recent years [51]. The evaluation of JCL plants that have been cultivated in southern areas of Iran shows that it yields under 17 months of cultivation, whereas the JCL yielding time is 24 months on average. Regarding this, an appropriate decision making tool is required to commercialize JCL cultivation and biodiesel production in Iran. This paper addresses this problem and proposes such decision making tool considering the real world assumptions.

\subsection{Input parameters}

As mentioned earlier, a number of parameters in the given problem are under epistemic uncertainty; therefore, trapezoidal fuzzy numbers are used to model their behavior. In this paper, available historical data are used in order to find the most possible values of the parameters. In addition, experts' opinions are used for determining the range of variation of fuzzy parameters. To make the fuzzy parameters, expert determines the variation percentage from the most possibilistic value a fuzzy parameter. All data used in this study will be provided on request.

The problem is considered for a 7-year planning horizon. Nevertheless, to decrease the computational 
complexity of the problem, each year is regarded as a time period. Thus, the overall planning horizon includes seven time periods. For estimating the most possible values of biodiesel demands, the demand of fossil-based diesel is predicted according to historical data. Subsequently, the demand of biodiesel is estimated according to the predetermined combination percentages of fossil-based diesel and biodiesel. In this case study, big cities, including Tehran, Razavi Khorasan, Tabriz, Isfahan, Khuzestan, Markazi, Fars, Ghom, Ghazvin, and Kerman are considered as biodiesel consumer centers. Table 1 shows the biodiesel demand for big provinces of Iran.

Table 1. Biodiesel demand in big cities (ton)

\begin{tabular}{cccccccc}
\hline \multirow{2}{*}{ Province } & 1 & 2 & 3 & 4 & 5 & 6 & 7 \\
\cline { 2 - 7 } Tabriz & 21200 & 21460 & 21720 & 54950 & 55600 & 84375 & 113800 \\
Isfahan & 66820 & 67640 & 68460 & 173200 & 175300 & 266175 & 359200 \\
Tehran & 73020 & 110850 & 112170 & 189200 & 287250 & 387800 & 392600 \\
Khorasan R. & 39220 & 39700 & 40180 & 101700 & 102950 & 156300 & 210900 \\
Khozestan & 51340 & 51980 & 52620 & 133150 & 134750 & 204525 & 275900 \\
Fars & 48240 & 48820 & 49400 & 124950 & 126450 & 192000 & 259200 \\
Gazvin & 23660 & 23940 & 24240 & 24540 & 37260 & 37710 & 63600 \\
Gom & 13660 & 13820 & 13980 & 14140 & 21450 & 21690 & 36550 \\
Kerman & 46320 & 46900 & 47480 & 48060 & 72960 & 73830 & 124500 \\
Markazi & 25180 & 25500 & 25820 & 26140 & 39690 & 40170 & 67750 \\
\hline
\end{tabular}

Glycerin is a byproduct of transesterification process and it can be used in the production of hygienic products. In Iran, 11 provinces have factories that produce hygienic products. However, Tehran produces $75 \%$ of hygienic products. In this study, it is assumed that the demand of glycerin in each province is consistent with the capacity of the factory producing hygienic products in that province. The amount of WCO production in each province is provided by Iranian Fuel Conversion Company (www.ifco.ir). Cost parameters are considered according to own calculations and the feasibility studies that have previously been performed in Iran. It should be mentioned that the calculated cost parameters vary among different provinces according to their total price index. Price index is the weighted average of the prices of a set of goods and services in a province, and therefore it can be used to compare costs in different provinces. These costs are also estimated according to average annual inflation rate in Iran in forthcoming years.

Transportation cost between two points is equal to the product of unitary transportation cost and the distance between them. Road and rail transportation modes are available for transporting JCL yields, WCO, and biodiesel between facilities. The road and rail transportation modes are available between 30 provinces and 11 provinces, respectively. Road and rail distances between two points are provided from Ministry of Roads \& Urban Development (www.mrud.ir) and Asia Seir Aras Company (www.asiaseiraras.com), respectively. It is assumed that JCL is converted to the JCL oil at the rate of 0.35. Also, JCL and WCO oils are converted to biodiesel at the rate of 0.83 . The most possible value of JCL harvest in different periods is provided from [3]. It is worth mentioning that JCL yields are between 2 to $12 \mathrm{t} / \mathrm{ha} / \mathrm{y}$ according to the literature. However, in this study, JCL yields are assumed to be between 2 to $7 \mathrm{t} / \mathrm{ha} / \mathrm{y}$ as we consider only arid and semi-arid areas as potential locations of JCL cultivation. The assumed amount of JCL yields in our study is based on scientific reports and real experiences [3,52]. 
On account of the fact that JCL can grow in special conditions, determining the potential locations for JCL cultivation is of great importance. Hence, it is required to determine these locations before designing the supply chain. Iran has 30 provinces that can be considered as the potential locations of JCL cultivation. Nonetheless, there are three features, including low soil $\mathrm{pH}$, long frost period, and waterlogging condition that prevent JCL growth. Consequently, the provinces with these features are removed from the potential locations of JCL cultivation. Among all provinces, Gilan and Mazandaran by reason of soil with low $\mathrm{pH}$ and waterlogging condition; Kurdistan, Ardabil, East Azerbaijan, and West Azerbaijan as a consequence of long frost period; and Golestan due of low soil $\mathrm{pH}$ are excluded from the potential locations. Afterwards, in order to measure the efficiency of the other provinces in cultivation of JCL, a Unified Data Envelopment Analysis (UDEA) model is utilized and the provinces with an efficiency greater than 0.8 are chosen as the potential locations of JCL cultivation. Accordingly, 9 provinces, including Khuzestan, Sistan and Baluchestan, Razavi Khorasan, Ghom, Kermanshah, Ilam, Bushehr, Chahar Mahal Bakhtiari, and Hormozgan are chosen as the potential locations for JCL cultivation. In the proposed model, three capacity levels have been considered to extend the capacity of oil extraction centers and bio-refineries in the upcoming periods. Also, six capacity levels have been considered for JCL cultivation areas.

\subsection{Results and discussion}

The proposed model is coded in GAMS 23.5 optimization software and solved by CPLEX solver. The model has 1,745 binary variables, 214,574 continuous variables, and 4,706 constraints. Feasibility degree of the constraints $(\alpha)$ is assumed to be 0.8 . Table 2 demonstrates the selected JCL supply centers and their capacities. Table 3 shows the selected provinces for establishing JCL oil extraction centers and their capacities. The input JCL in oil extraction centers is provided from internal JCL supply centers or external suppliers. In Table 3, total capacity indicates the sum of initial capacity and capacity expansion in different periods. According to the results obtained from the proposed model, the import of JCL is needed only in the first and last periods. Total JCL required in the first period and last period are 159,612 tons and 257,969 tons, respectively. It is worth mentioning that JCL yields are low in the first period. Also, in the last period, it is required to import JCL. Most of the oil extraction centers will have the same capacity; however, the established oil extraction centers in Ghom and South Khorasan provinces have a lower capacity. In addition, the number of JCL oil extraction centers is more than that of JCL supply centers, which results from decreasing total transportation costs in the given supply chain. Indeed, the proposed model decreases the total transportation costs through establishing more facilities. Table 4 demonstrates the location and capacity of bio-refineries. In Table 4, total capacity equals to the sum of initial capacity and capacity expansion in different periods. Also, bio-refinery capacity expansion only occurs in Isfahan. According to the results, the bio-refinery in Isfahan needs a capacity expansion equal to 160,000 tons in the first period.

Table 2. Location and capacity of JCL supply centers

\begin{tabular}{cc}
\hline Province & Capacity (ha) \\
\hline Ilam & 110000 \\
Bushehr & 70000 \\
Chahar Mahal Bakhtiari & 110000 \\
Razavi Khorasan & 130000 \\
Khuzestan & 90000 \\
Sistan and Baluchestan & 110000 \\
Ghom & 70000 \\
\hline
\end{tabular}




\begin{tabular}{cc}
\hline Kermanshah & 110000 \\
Hormozgan & 70000 \\
\hline
\end{tabular}

Table 3. Location and capacity of oil extraction centers

\begin{tabular}{cc}
\hline Province & Total capacity (ton) \\
\hline Ilam & 600000 \\
Bushehr & 600000 \\
Chahar Mahal Bakhtiari & 600000 \\
South Khorasan & 400000 \\
Razavi Khorasan & 600000 \\
Khuzestan & 600000 \\
Sistan and Baluchestan & 600000 \\
Ghom & 400000 \\
Kermanshah & 600000 \\
Hormozgan & 600000 \\
\hline Table 4. Location and capacity of bio-refineries \\
\hline Province & Total capacity (tons) \\
\hline Isfahan & 440000 \\
Bushehr & 440000 \\
South Khorasan & 360000 \\
Razavi Khorasan & 280000 \\
Khuzestan & 280000 \\
Kermanshah & 440000 \\
\hline
\end{tabular}

Fig. 2 shows the optimal structure of the given supply chain in the case study. According to Fig. 2, it can be concluded that the optimal supply chain has a decentralized structure. The optimal supply chain is more centralized in the cultivation areas in comparison to the other areas. That is because the model prefers to reduce the distance between JCL supply centers and bio-refineries so that the transportation costs may be decreased. 


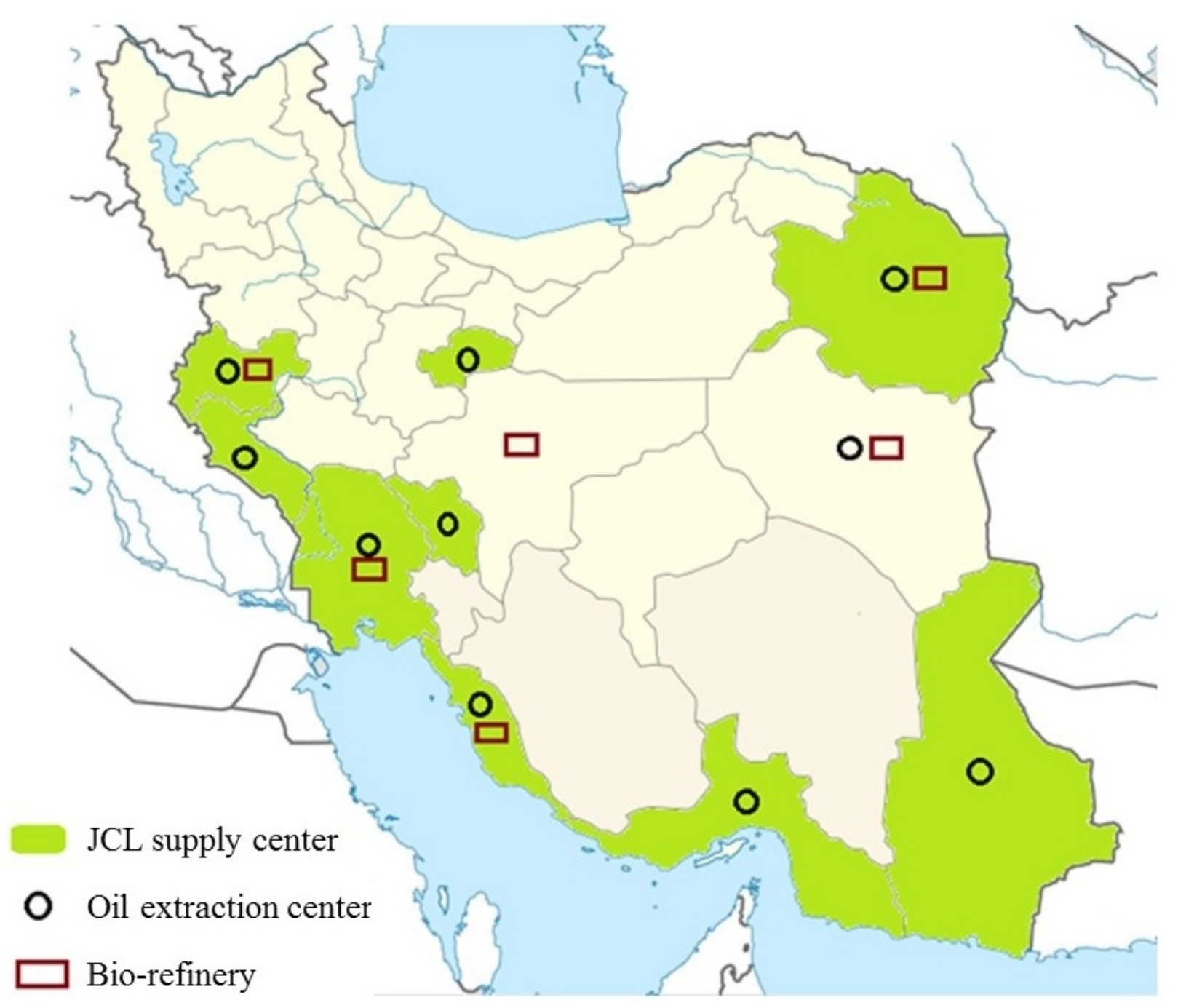

Fig. 2. The illustration of the second-generation biodiesel supply chain in Iran

To validate the results of the proposed credibility-based fuzzy chance constrained programming approach, its main results are compared with those of simple deterministic model. Table 5 illustrates the construction variables (i.e., binary variables) of biodiesel supply chain network achieved by deterministic model. It should be noted that optimality gap in deterministic model was set to 0.05 .

Table 5. Results of construction variables achieved by deterministic model

\begin{tabular}{ccc}
\hline JCL supply center & Oil extraction center & Biorefinery \\
\hline Ilam & Ilam & Isfahan \\
Bushehr & Bushehr & Ilam \\
Khuzestan & Khorasan J. & Khorasan J. \\
Chahar Mahal Bakhtiari & Chahar Mahaal and Bakhtiari & Bushehr \\
Sistan and Baluchestan & Khorasan R. & Khozestan \\
Kermanshah & Khorasan Sh. & Kermanshah \\
Hormozgan & Khozestan & Hormozgan \\
\hline
\end{tabular}


Comparing Table 5 with Tables 2, 3, and 4 shows that some locations selected for constructing biodiesel supply chain network by the two models are similar. However, the proposed credibility-based fuzzy chance constrained programming approach selects more locations than deterministic one to deal with epistemic uncertainty. Although, more locations will impose higher construction costs, it will avoid infeasibility of the model and save costs when realization of uncertain parameters [10].

\subsubsection{Results of BDA and ABDA for solving the propose model}

To evaluate and analyze the performance of the developed ABDA, both BDA and ABDA are coded in GAMS 23.5 optimization software. Figs. 3 and 4 show the convergence of lower and upper bounds using BDA and ABDA, respectively. $\varepsilon$ is assumed to be 0.01 . According to the results, ABDA converges on the optimal solution remarkably faster than BDA does. By the ABDA, the lower and upper bounds are achieved equal to 1148090000 and 1158800000 , Respectively. Also, by the BDA, the lower and upper bounds are calculated equal to 1143910000 and 1152810000 , respectively. Therefore, considering the large number for objective function value, the absolute gaps calculated by the BDA and ABDA are reasonable. Although we have set the relative gap equal to 0.01, the relative gaps achieved by ABDA and BDA are equal to 0.009 and 0.007 , respectively.

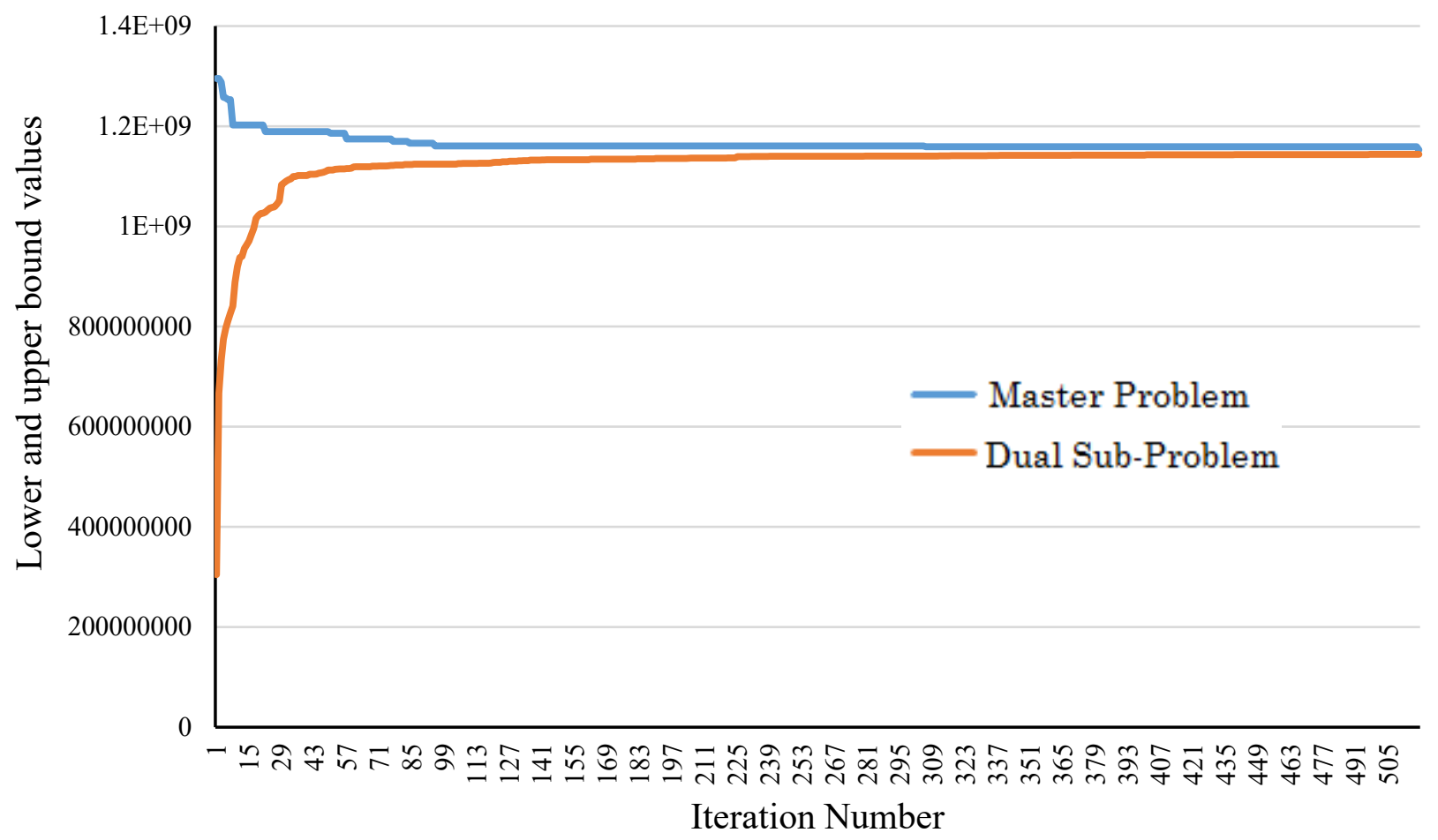

Fig. 3. The convergence progression of the proposed BDA 


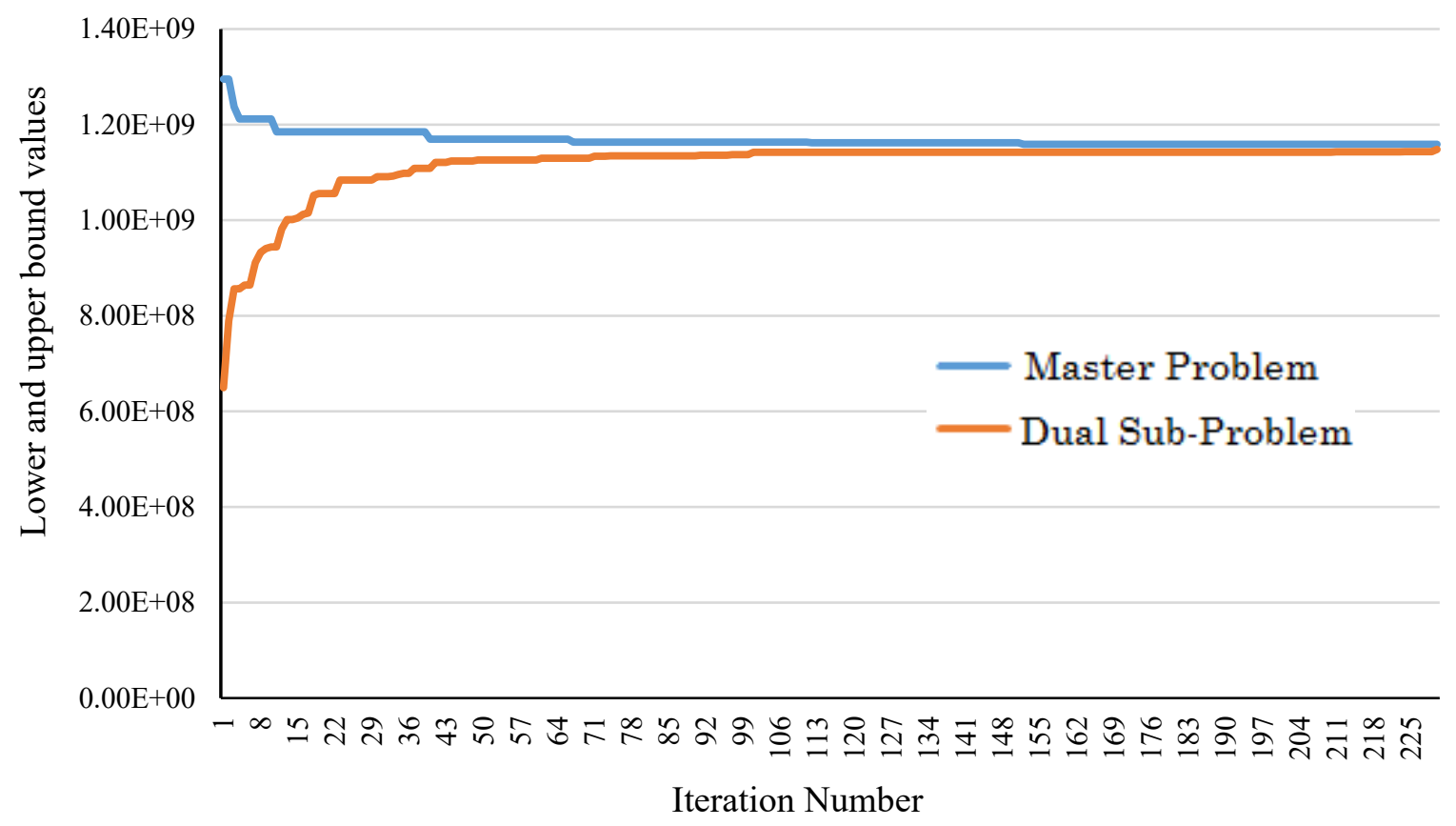

Fig. 4. The convergence progression of the proposed ABDA

ABDA converges on the optimal solution under 1.24 hours by performing 230 iterations, while BDA converges on the optimal solution under 14.36 hours by performing 518 iterations. This considerable difference between the BDA and ABDA is made after adding local branching cut to MP. Indeed, this local branching cut not only makes performing each iteration faster, but it also decreases the number of required iterations for obtaining the optimal solution. ABDA provides us with better lower and upper bounds in comparison with BDA. Table 6 compares the values of lower and upper bounds of BDA and ABDA for the iterations 1 to 30 .

It should be noted that solving the full-space model with CPLEX solver of the GAMS reaches to error "OUT OF MEMORY" after $1.22 \mathrm{~h}$ at gap $17 \%$. Therefore, the solution is not achieved by solver CPLEX in full-space mode.

Table 6. Comparison of the upper and lower bounds of ABDA and BDA

\begin{tabular}{cccccc}
\hline \multirow{2}{*}{$\begin{array}{c}\text { Iteration } \\
\text { number }\end{array}$} & \multicolumn{2}{c}{ ABDA } & & \multicolumn{2}{c}{ BDA } \\
\cline { 2 - 3 } \cline { 5 - 6 } & bound & $\begin{array}{c}\text { Lower } \\
\text { bound }\end{array}$ & & $\begin{array}{c}\text { Upper } \\
\text { bound }\end{array}$ & $\begin{array}{c}\text { Lower } \\
\text { bound }\end{array}$ \\
\hline 1 & 1295300000 & 649572000 & & 1295300000 & 304650000 \\
2 & 1295300000 & 789579000 & & 1295300000 & 666933000 \\
3 & 1237150000 & 856470000 & & 1288040000 & 732936000 \\
4 & 1212080000 & 856470000 & & 1258000000 & 774810000 \\
5 & 1212080000 & 864317000 & & 1256310000 & 797088000 \\
6 & 1212080000 & 864317000 & & 1253240000 & 812282000 \\
7 & 1212080000 & 911250000 & & 1253240000 & 827028000 \\
8 & 1212080000 & 932853000 & & 1202560000 & 840955000 \\
\hline
\end{tabular}




\begin{tabular}{rrrrrr}
\hline \multirow{2}{*}{$\begin{array}{c}\text { Iteration } \\
\text { number }\end{array}$} & $\begin{array}{c}\text { Upper } \\
\text { bound }\end{array}$ & $\begin{array}{c}\text { Lower } \\
\text { bound }\end{array}$ & & $\begin{array}{c}\text { Bpper } \\
\text { bound }\end{array}$ & $\begin{array}{c}\text { Lower } \\
\text { bound }\end{array}$ \\
\cline { 2 - 5 } \cline { 5 - 6 } & 1212080000 & 940910000 & & 1202560000 & 888632000 \\
10 & 1212080000 & 943889000 & & 1202560000 & 919593000 \\
11 & 1184870000 & 943889000 & & 1202560000 & 937880000 \\
12 & 1184870000 & 981519000 & & 1202560000 & 939962000 \\
13 & 1184870000 & 1001320000 & & 1202560000 & 955749000 \\
14 & 1184870000 & 1001320000 & & 1202560000 & 963600000 \\
15 & 1184870000 & 1004730000 & & 1202560000 & 970838000 \\
16 & 1184870000 & 1012350000 & & 1202560000 & 983838000 \\
17 & 1184870000 & 1014850000 & & 1202560000 & 996253000 \\
18 & 1184870000 & 1052190000 & & 1202560000 & 1015900000 \\
19 & 1184870000 & 1055630000 & & 1202560000 & 1021980000 \\
20 & 1184870000 & 1055630000 & & 1202560000 & 1026060000 \\
21 & 1184870000 & 1055630000 & & 1202560000 & 1026150000 \\
22 & 1184870000 & 1055630000 & & 1189060000 & 1028450000 \\
23 & 1184870000 & 1083820000 & & 1189060000 & 1032600000 \\
24 & 1184870000 & 1083820000 & & 1189060000 & 1036580000 \\
25 & 1184870000 & 1083820000 & 1189060000 & 1037870000 \\
26 & 1184870000 & 1083820000 & 1189060000 & 1039160000 \\
27 & 1184870000 & 1083820000 & & 1189060000 & 1044510000 \\
28 & 1184870000 & 1083820000 & & 1189060000 & 1050670000 \\
29 & 1184870000 & 1083820000 & & 1189060000 & 1083330000 \\
30 & 1184870000 & 1091020000 & & 1189060000 & 1086760000 \\
\hline
\end{tabular}

\section{Conclusions and future research directions}

This paper presents a possibilistic programming model for designing a second-generation biodiesel supply chain network under epistemic uncertainty of input parameters. The given problem is a multi-feedstock, multi-product, multi-period, multi-modal supply chain network design problem. The proposed model determines the main tactical and strategic decisions, including optimal sourcing and allocation of JCL seeds and WCO; the capacity and location of JCL supply centers, oil extraction centers, bio-refineries; the capacity expansion of oil extraction centers and bio-refineries; the amount of imported JCL seeds to oil extraction centers; the amount of produced biodiesel and glycerin at bio-refineries; the amount of produced JCL oil at oil extraction centers; the material flow between the supply chain stages; and transportation modes in different periods so much so that the total costs of the supply chain is minimized. To deal with the epistemic uncertainty of the parameters, a credibility-based possibilistic programming approach is employed to convert the original possibilistic programming model into a crisp counterpart. An ABDA using efficient acceleration mechanisms is devised to deal with the computational complexity of solving the proposed model in an efficient manner. The performance of the proposed possibilistic programming model as well as the efficiency of the developed ABDA are validated by performing a computational analysis 
using a real case study in Iran. According to the results obtained from the proposed model, the import of JCL is needed only in the first and last periods. Most of the oil extraction centers will have the same capacity; however, the established oil extraction centers in Ghom and South Khorasan provinces have a lower capacity. The proposed model reduces the total transportation costs through establishing more facilities. Also, bio-refinery capacity expansion only occurs in Isfahan. Also, according to the results, ABDA converges on the optimal solution remarkably faster than BDA does.

Quite a few extensions on this work could be done for future research. Different types of uncertainty for uncertain parameters can be considered. Also, environmental and social impacts of supply chain network design decisions could be addressed. Additionally, the presented approach could be employed for dealing with the uncertainty in bioethanol supply chains. The proposed approach could be integrated with stochastic programming methods to handle the randomness and epistemic uncertainty, simultaneously.

\section{Acknowledgement}

The first author appreciates for the Iran National Science Foundation (INSF) for the financial support of this study. Also, the authors thank the anonymous referees for their valuable comments.

\section{Appendix A.}

Forming MP and DSP for the proposed possibilistic programming model

In order to form DSP, first, the integer variables of the proposed possibilistic programming model are fixed. In the proposed model, capacity variables that are a product of a parameter and a binary variable are converted to an integer variable. The following model is PSP in which the binary and integer variables have been fixed. It should be noted that when an integer variable is fixed, its corresponding terms are removed from the objective function and the constraints.

$$
\begin{aligned}
& \text { Min } Z 1=\sum_{f, l, i, t}\left[\frac{J T_{f l i t}^{(1)}+J T_{f l i t}^{(2)}+J T_{\text {flit }}^{(3)}+J T_{\text {flit }}^{(4)}}{4}\right] \cdot J a_{f l i t} \\
& +\sum_{i, l, j, t}\left[\frac{P O_{i t}^{(1)}+P O_{i t}^{(2)}+P O_{i t}^{(3)}+P O_{i t}^{(4)}}{4}+\frac{\left.O T_{i l j t}^{(1)}+O T_{i l j t}^{(2)}+O T_{i l j t}^{(3)}+O T_{i l j t}^{(4)}\right]}{4}\right] \cdot O_{i l j t} \\
& +\sum_{g, l, j, t}\left[\frac{W T_{g l j t}^{(1)}+W T_{g l j t}^{(2)}+W T_{g l j t}^{(3)}+W T_{g l j t}^{(4)}}{4}\right] \cdot W_{g l j t} \\
& +\sum_{j, l, k, t}\left[\frac{P B_{j t}^{(1)}+P B_{j t}^{(2)}+P B_{j t}^{(3)}+P B_{j t}^{(4)}}{4}\right] \\
& \left.+\frac{B T_{j l k t}^{(1)}+B T_{j l k t}^{(2)}+B T_{j l k t}^{(3)}+B T_{j l k t}^{(4)}}{4}\right] \cdot B_{j l k t} \\
& +\sum_{i, t}\left[\frac{P I_{i t}^{(1)}+P I_{i t}^{(2)}+P I_{i t}^{(3)}+P I_{i t}^{(4)}}{4}\right] \cdot I m_{i t}
\end{aligned}
$$




$$
-\sum_{j, t}\left[\frac{P G_{j t}^{(1)}+P G_{j t}^{(2)}+P G_{j t}^{(3)}+P G_{j t}^{(4)}}{4}\right] \cdot G L_{j t}
$$

Subject to:

$$
\begin{aligned}
& \sum_{j, l} B_{j l k t} \geq\left(2-2 \alpha_{k t}\right) D_{k t}^{(3)}+\left(2 \alpha_{k t}-1\right) D_{k t}^{(4)} \quad \forall k, t \quad \psi 1_{k t} \\
& \sum_{l, j} W_{g l j t} \geq W O_{g t}^{(2)} \quad \forall g, t \quad \psi 2_{g t} \\
& -\sum_{l, j} W_{g l j t} \geq-W O_{g t}^{(3)} \quad \forall g, t \quad \psi 3_{g t} \\
& \sum_{l, i} J a_{f l i t} \geq \eta_{f t}^{(2)} \sum_{p} C J_{f}^{p} \cdot \bar{x}_{f}^{p} \quad \forall f, t \quad \psi \psi 4_{f t} \\
& -\sum_{l, i} J a_{f l i t} \geq-\eta_{f t}^{(3)} \sum_{p} C J_{f}^{p} \cdot \bar{x}_{f}^{p} \quad \forall f, t \quad \psi 5_{f t} \\
& \sum_{l, j} O_{i l j t}-\varphi \times\left(\sum_{f, l} J a_{f l i t}+I m_{i t}\right)=0 \quad \forall i, t \quad \psi 6_{i t} \\
& \sum_{l, k} B_{j l k t}-\beta \times\left(\sum_{g, l} W_{g l j t}+\sum_{i, l} O_{i l j t}\right)=0 \quad \forall j, t \quad \psi 7_{j t} \\
& G L_{j t}-(1-\beta) \times\left(\sum_{g, l} W_{g l j t}+\sum_{i, l} O_{i l j t}\right)=0 \quad \forall j, t \quad \psi 8_{j t} \\
& -I m_{i t} \geq-M I_{i t} \quad \forall i, t \quad \psi 9_{i t} \\
& -\sum_{f, l} J a_{f l i t}-I m_{i t} \geq-\overline{C O}_{i t} \quad \forall i, t \quad \psi \psi 10_{i t} \\
& -\sum_{g, l} W_{g l j t}-\sum_{i, l} o_{i l j t} \geq-\overline{C B}_{j t} \quad \forall j, t \quad \psi 11_{j t}
\end{aligned}
$$

In order to formulate the dual form of the above PSP, the variables $\psi 1$ to $\psi 11$ related to the DSP are defined according to the right-hand-side values of the constraints of PSP. It is worth mentioning that PSP is a minimization problem, and therefore the variables $\psi 6, \psi 7, \psi 8$ are free and the other variables are non-negative. The variables with a bar sign above them have been fixed in the PSP. According to the above descriptions, the DSP is presented as follows.

$$
\begin{aligned}
\operatorname{Max} \quad D Z & =\sum_{k, t}\left(\left(2-2 \alpha_{k t}\right) D_{k t}^{(3)}+\left(2 \alpha_{k t}-1\right) D_{k t}^{(4)}\right) \psi 1_{k t}+\sum_{g, t} W O_{g t}^{(2)} \cdot \psi 2_{g t} \\
& -\sum_{g, t} W O_{g t}^{(3)} \cdot \psi 3_{g t}+\sum_{p, f, t} \eta_{f t}^{(2)} C J_{f}^{p} \cdot \bar{x}_{f}^{p} \cdot \psi 4_{f t}-\sum_{p, f, t} \eta_{f t}^{(3)} C J_{f}^{p} \cdot \bar{x}_{f}^{p} \cdot \psi 5_{f t}
\end{aligned}
$$




$$
-\sum_{i, t} M I_{i t} \cdot \psi 9_{i t}-\sum_{i, t} \overline{C O}_{i t} \cdot \psi 10_{i t}-\sum_{j, t} \overline{C B}_{j t} \cdot \psi 11_{j t}
$$

S.t.

$$
\begin{aligned}
& \psi 1_{k t}-\psi 7_{j t} \leq\left[\frac{P B_{j t}^{(1)}+P B_{j t}^{(2)}+P B_{j t}^{(3)}+P B_{j t}^{(4)}}{4}\right. \\
& \left.+\frac{B T_{j l k t}^{(1)}+B T_{j l k t}^{(2)}+B T_{j l k t}^{(3)}+B T_{j l k t}^{(4)}}{4}\right] \quad \forall j, l, k, t \\
& \psi 2_{g t}-\psi 3_{g t}-\beta \cdot \psi 7_{j t}-(1-\beta) \cdot \psi 8_{j t}-\psi 11_{j t} \\
& \leq\left[\frac{W T_{\text {gljt }}^{(1)}+W T_{\text {gljt }}^{(2)}+W T_{\text {gljt }}^{(3)}+W T_{\text {gljt }}^{(4)}}{4}\right] \quad \forall g, l, j, t \\
& \psi 4_{f t}-\psi 5_{f t}-\varphi \cdot \psi 6_{i t}-\psi 10_{i t} \leq\left[\frac{J T_{\text {flit }}^{(1)}+J T_{\text {flit }}^{(2)}+J T_{\text {flit }}^{(3)}+J T_{\text {flit }}^{(4)}}{4}\right] \quad \forall f, l, i, t \\
& -\varphi . \psi 6_{i t}-\psi 9_{i t}-\psi 10_{i t} \leq\left[\frac{P I_{i t}^{(1)}+P I_{i t}^{(2)}+P I_{i t}^{(3)}+P I_{i t}^{(4)}}{4}\right] \quad \forall i, t \\
& \psi 6_{i t}-\beta . \psi 7_{j t}-(1-\beta) \cdot \psi 8_{j t}-\psi 11_{j t} \\
& \leq\left[\frac{P O_{i t}^{(1)}+P O_{i t}^{(2)}+P O_{i t}^{(3)}+P O_{i t}^{(4)}}{4}\right. \\
& \left.+\frac{O T_{i l j t}^{(1)}+O T_{i l j t}^{(2)}+O T_{i l j t}^{(3)}+O T_{i l j t}^{(4)}}{4}\right] \quad \forall i, l, j, t \\
& \psi 8_{j t} \leq-\left[\frac{P G_{j t}^{(1)}+P G_{j t}^{(2)}+P G_{j t}^{(3)}+P G_{j t}^{(4)}}{4}\right] \quad \forall j, t \\
& \psi 6_{i t}, \psi 7_{j t}, \psi 8_{j t} \text { are free variables } \\
& \psi 1-\psi 5 \text { and } \psi 9-\psi 11 \geq 0
\end{aligned}
$$

Regarding the structure of the proposed model, some valid inequalities can be added to the MP that restrict the feasible region and guarantee the feasibility of the solutions, and therefore the DSP in the next iteration is bounded, feasible, and with at least one optimal solution [38]. Since the DSP is always bounded and feasible for any binary variables that satisfy constraint, there is no need to add feasibility cuts to MP, and in each iteration only optimality cuts would be added. In spite of having useful information from the original problem and producing feasible solutions, the valid inequalities should have a low impact on the complexity of the MP. According to the structure of the proposed model, three valid inequalities (constraints) can be added to MP as follows:

$$
\begin{array}{ll}
\beta . \sum_{j} C B_{j t} \geq \sum_{k}\left(\left(2-2 \alpha_{k t}\right) D_{k t}^{(3)}+\left(2 \alpha_{k t}-1\right) D_{k t}^{(4)}\right) & \forall t \\
\beta \cdot\left(\varphi \cdot \sum_{i} C O_{i t}+\sum_{g} W O_{g t}^{(3)}\right) \geq \sum_{k}\left(\left(2-2 \alpha_{k t}\right) D_{k t}^{(3)}+\left(2 \alpha_{k t}-1\right) D_{k t}^{(4)}\right) & \forall t
\end{array}
$$




$$
\begin{gathered}
\beta \cdot \varphi \cdot\left(\sum_{f, p} \eta_{f t}^{(3)} C J_{f}^{p} \cdot x_{f}^{p}+\sum_{i} M I_{i t}\right)+\beta \cdot \sum_{g} W O_{g t}^{(3)} \\
\geq \sum_{k}\left(\left(2-2 \alpha_{k t}\right) D_{k t}^{(3)}+\left(2 \alpha_{k t}-1\right) D_{k t}^{(4)}\right) \quad \forall t
\end{gathered}
$$

Constraints (A.22) ensures that the product of the conversion factor of JCL oil to biodiesel and the capacity of the established bio-refineries must be at least equal to biodiesel demand. In order to form constraint (A.23), suppose that the bio-refineries do not exist and both JCL oil and biodiesel are produced at oil extraction centers. With this in mind, the sum of WCO and capacity multiplied by conversion rates is at least equal to biodiesel demand. Similarly, in order to form the constraint (A.24), suppose bio-refineries and oil extraction centers do not exist, and all biodiesel demand is supplied by JCL supply centers. Above constraints prevent the model from establishing insufficient facilities, restrict solution space, and therefore improve the quality of MP solutions. In each iteration of BDA, the solution obtained from the above DSP and the cuts obtained from it are used in the MP problem that is presented as follows.

$$
\begin{aligned}
\operatorname{Min} Z & =\xi+\sum_{f, p}\left[\frac{F J_{f}^{p(1)}+F J_{f}^{p(2)}+F J_{f}^{p(3)}+F J_{f}^{p(4)}}{4}\right] \cdot x_{f}^{p} \\
& +\sum_{i, t}\left[\frac{F O_{i t}^{(1)}+F O_{i t}^{(2)}+F O_{i t}^{(3)}+F O_{i t}^{(4)}}{4}\right] \cdot\left(y_{i t}-y_{i(t-1)}\right) \\
& +\sum_{j, t}\left[\frac{F B_{j t}^{(1)}+F B_{j t}^{(2)}+F B_{j t}^{(3)}+F B_{j t}^{(4)}}{4}\right] \cdot\left(u_{j t}-u_{j(t-1)}\right) \\
& +\sum_{i, t, q}\left[\frac{V O_{i t}^{q(1)}+V O_{i t}^{q(2)}+V O_{i t}^{q(3)}+V O_{i t}^{q(4)}}{4}\right] \cdot y e_{i t}^{q} \\
& +\sum_{j, t}\left[\frac{V B_{j t}^{s(1)}+V B_{j t}^{s(2)}+V B_{j t}^{s(3)}+V B_{j t}^{s(4)}}{4}\right] \cdot u e_{j t}^{s}
\end{aligned}
$$

s.t.

$$
\begin{aligned}
& \xi \geq \sum_{k, t}\left(\left(2-2 \alpha_{k t}\right) D_{k t}^{(3)}+\left(2 \alpha_{k t}-1\right) D_{k t}^{(4)}\right) \bar{\psi} 1_{k t}+\sum_{g, t} W O_{g t}^{(2)} \cdot \bar{\psi} 2_{g t} \\
& -\sum_{g, t} W O_{g t}^{(3)} \cdot \bar{\psi} 3_{g t}+\sum_{p, f, t} \eta_{f t}^{(2)} C J_{f}^{p} \cdot x_{f}^{p} \cdot \bar{\psi} 4_{f t}-\sum_{p, f, t} \eta_{f t}^{(3)} C J_{f}^{p} \cdot x_{f}^{p} \cdot \bar{\psi} 5_{f t} \\
& -\sum_{i, t} M I_{i t} \cdot \bar{\psi} 9_{i t}-\sum_{i, t} C O_{i t} \cdot \bar{\psi} 10_{i t}-\sum_{j, t} C B_{j t} \cdot \bar{\psi} 11_{j t} \\
& y_{i t} \leq y_{i(t+1)} \quad \forall i, t \\
& u_{j t} \leq u_{j(t+1)} \quad \forall j, t \\
& y e_{i t}^{q} \leq y_{i t} \quad \forall i, t, q \\
& u e_{j t}^{s} \leq u_{j t} \quad \forall j, t, s \\
& \sum_{q} y e_{i t}^{q} \leq 1 \quad \forall i, t
\end{aligned}
$$




$$
\begin{aligned}
& \sum_{s} u e_{j t}^{s} \leq 1 \quad \forall j, t \\
& \sum_{p} x_{f}^{p} \leq 1 \quad \forall f \\
& \sum_{i, t}\left(y_{i t}-y_{i(t-1)}\right) \leq \operatorname{Max} y \\
& \sum_{j, t}\left(u_{j t}-u_{j(t-1)}\right) \leq \operatorname{Maxu} \\
& \sum_{q, t} y e_{i t}^{q} \cdot C E O_{i t}^{q} \leq U O_{i} \quad \forall i \\
& \sum_{s, t} u e_{j t}^{s} . C E B_{j t}^{S} \leq U B_{j} \quad \forall j \\
& C O_{i t}=C O_{i(t-1)}+I C O_{i t} \cdot\left(y_{i t}-y_{i(t-1)}\right)+\sum_{q} y e_{i t}^{q} \cdot C E O_{i t}^{q} \quad \forall i, t \\
& C B_{j t}=C B_{j(t-1)}+I C B_{j t} \cdot\left(u_{j t}-u_{j(t-1)}\right)+\sum_{s} u e_{j t}^{s} \cdot C E B_{j t}^{S} \quad \forall j, t \\
& \beta . \sum_{j} C B_{j t} \geq \sum_{k}\left(\left(2-2 \alpha_{k t}\right) D_{k t}^{(3)}+\left(2 \alpha_{k t}-1\right) D_{k t}^{(4)}\right) \quad \forall t \\
& \beta \cdot\left(\varphi \cdot \sum_{i} C O_{i t}+\sum_{g} W O_{g t}^{(3)}\right) \geq \sum_{k}\left(\left(2-2 \alpha_{k t}\right) D_{k t}^{(3)}+\left(2 \alpha_{k t}-1\right) D_{k t}^{(4)}\right) \quad \forall t \\
& \beta . \varphi \cdot\left(\sum_{f, p} \eta_{f t}^{(3)} C J_{f}^{p} \cdot x_{f}^{p}+\sum_{i} M I_{i t}\right)+\beta \cdot \sum_{g} W O_{g t}^{(3)} \\
& \geq \sum_{k}\left(\left(2-2 \alpha_{k t}\right) D_{k t}^{(3)}+\left(2 \alpha_{k t}-1\right) D_{k t}^{(4)}\right) \quad \forall t \\
& x_{f}^{p}, y_{i t}^{q}, u_{j t}^{s}, y e_{i t}^{q}, u e_{j t}^{s} \in\{0,1\}, \quad \xi \geq 0
\end{aligned}
$$

Where $\xi$ is a non-negative dummy variable. According to the above descriptions, only optimality cut and valid inequalities are added to the MP.

\section{References}

1. Babazadeh, R., Optimal design and planning of biodiesel supply chain considering non-edible feedstock. Renewable and Sustainable Energy Reviews, 2017. 75: p. 1089-1100. 
2. Pandey, V.C., et al., Jatropha curcas: A potential biofuel plant for sustainable environmental development. Renewable and Sustainable Energy Reviews, 2012. 16(5): p. 2870-2883.

3. Achten, W., et al., Jatropha bio-diesel production and use. Biomass and bioenergy, 2008. 32(12): p. 1063-1084.

4. Babazadeh, R., et al., An integrated data envelopment analysis-mathematical programming approach to strategic biodiesel supply chain network design problem. Journal of Cleaner Production, 2017. 147: p. 694-707.

5. Ghaderi, H., M.S. Pishvaee, and A. Moini, Biomass supply chain network design: an optimization-oriented review and analysis. Industrial crops and products, 2016. 94: p. 972-1000.

6. Ren, J., et al., Life cycle cost optimization of biofuel supply chains under uncertainties based on interval linear programming. Bioresource technology, 2015. 187: p. 6-13.

7. Lim, C.H. and H.L. Lam, Biomass supply chain optimisation via novel biomass element life cycle analysis (BELCA). Applied Energy, 2016. 161: p. 733-745.

8. Bairamzadeh, S., M.S. Pishvaee, and M. Saidi-Mehrabad, Multiobjective Robust Possibilistic Programming Approach to Sustainable Bioethanol Supply Chain Design under Multiple Uncertainties. Industrial \& Engineering Chemistry Research, 2015. 55(1): p. 237-256.

9. Mohseni, S., M.S. Pishvaee, and H. Sahebi, Robust design and planning of microalgae biomassto-biodiesel supply chain: A case study in Iran. Energy, 2016. 111: p. 736-755.

10. Cambero, C. and T. Sowlati, Incorporating social benefits in multi-objective optimization of forest-based bioenergy and biofuel supply chains. Applied Energy, 2016. 178: p. 721-735.

11. Bai, Y., Y. Ouyang, and J.-S. Pang, Enhanced models and improved solution for competitive biofuel supply chain design under land use constraints. European Journal of Operational Research, 2016. 249(1): p. 281-297.

12. Babazadeh, R., et al., A sustainable second-generation biodiesel supply chain network design problem under risk. Omega, 2017. 66: p. 258-277.

13. Grigoroudis, E., K. Petridis, and G. Arabatzis, RDEA: A recursive DEA based algorithm for the optimal design of biomass supply chain networks. Renewable Energy, 2014. 71: p. 113-122.

14. Miret, C., et al., Design of bioethanol green supply chain: Comparison between first and second generation biomass concerning economic, environmental and social criteria. Computers \& Chemical Engineering, 2016. 85: p. 16-35.

15. Roni, M.S., et al., A multi-objective, hub-and-spoke model to design and manage biofuel supply chains. Annals of Operations Research, 2016: p. 1-30.

16. Vera, D., et al., A Honey Bee Foraging approach for optimal location of a biomass power plant. Applied Energy, 2010. 87(7): p. 2119-2127.

17. Oberkampf, W.L., et al., Error and uncertainty in modeling and simulation. Reliability Engineering \& System Safety, 2002. 75(3): p. 333-357.

18. Oberkampf, W.L. and J.C. Helton, Evidence theory for engineering applications, in Engineering design reliability handbook. 2004, CRC Press. p. 197-226.

19. Pishvaee, M.S. and S.A. Torabi, A possibilistic programming approach for closed-loop supply chain network design under uncertainty. Fuzzy sets and systems, 2010. 161(20): p. 2668-2683.

20. Azadeh, A., H.V. Arani, and H. Dashti, A stochastic programming approach towards optimization of biofuel supply chain. Energy, 2014. 76: p. 513-525.

21. Li, Q. and G. Hu, Supply chain design under uncertainty for advanced biofuel production based on bio-oil gasification. Energy, 2014. 74: p. 576-584.

22. Osmani, A. and J. Zhang, Multi-period stochastic optimization of a sustainable multi-feedstock second generation bioethanol supply chain-A logistic case study in Midwestern United States. Land Use Policy, 2017. 61: p. 420-450.

23. Gitinavard, H., H. Ghaderi, and M.S. Pishvaee, Green supplier evaluation in manufacturing systems: a novel interval-valued hesitant fuzzy group outranking approach. Soft Computing, 2017. 
24. Tong, K., et al., Optimal design of advanced drop-in hydrocarbon biofuel supply chain integrating with existing petroleum refineries under uncertainty. biomass and bioenergy, 2014. 60: p. 108-120.

25. Ghaderi, H., A. Moini, and M.S. Pishvaee, A multi-objective robust possibilistic programming approach to sustainable switchgrass-based bioethanol supply chain network design. Journal of Cleaner Production, 2018.

26. Ahn, Y.-C., et al., Strategic planning design of microalgae biomass-to-biodiesel supply chain network: Multi-period deterministic model. Applied Energy, 2015. 154: p. 528-542.

27. Paulo, H., et al., Supply chain optimization of residual forestry biomass for bioenergy production: the case study of Portugal. Biomass and Bioenergy, 2015. 83: p. 245-256.

28. d'Amore, F. and F. Bezzo, Strategic optimisation of biomass-based energy supply chains for sustainable mobility. Computers \& Chemical Engineering, 2016. 87: p. 68-81.

29. Hombach, L.E., et al., Optimal design of supply chains for second generation biofuels incorporating European biofuel regulations. Journal of Cleaner Production, 2016. 133: p. 565575.

30. Yue, D., et al., Sustainable design and operation of cellulosic bioelectricity supply chain networks with life cycle economic, environmental, and social optimization. Industrial \& Engineering Chemistry Research, 2014. 53(10): p. 4008-4029.

31. Duarte, A., W. Sarache, and Y. Costa, Biofuel supply chain design from Coffee Cut Stem under environmental analysis. Energy, 2016. 100: p. 321-331.

32. Zhang, Y. and Y. Jiang, Robust optimization on sustainable biodiesel supply chain produced from waste cooking oil under price uncertainty. Waste Management, 2017. 60(Supplement C): p. 329339.

33. Ghaderi, H., M. Asadi, and S. Shavalpour, A switchgrass-based bioethanol supply chain network design model under auto-regressive moving average demand. Journal of Renewable Energy and Environment, 2016. 3(3): p. 1.

34. Mohseni, S. and M.S. Pishvaee, A robust programming approach towards design and optimization of microalgae-based biofuel supply chain. Computers \& Industrial Engineering, 2016. 100: p. 58-71.

35. Bairamzadeh, S., M. Saidi-Mehrabad, and M.S. Pishvaee, Modelling different types of uncertainty in biofuel supply network design and planning: A robust optimization approach. Renewable Energy, 2018. 116(Part A): p. 500-517.

36. Maheshwari, P., S. Singla, and Y. Shastri, Resiliency optimization of biomass to biofuel supply chain incorporating regional biomass pre-processing depots. Biomass and Bioenergy, 2017. 97(Supplement C): p. 116-131.

37. $\mathrm{Li}, \mathrm{X}$. and B. Liu, A sufficient and necessary condition for credibility measures. International Journal of Uncertainty, Fuzziness and Knowledge-Based Systems, 2006. 14(05): p. 527-535.

38. Pishvaee, M., J. Razmi, and S. Torabi, An accelerated Benders decomposition algorithm for sustainable supply chain network design under uncertainty: A case study of medical needle and syringe supply chain. Transportation Research Part E: Logistics and Transportation Review, 2014. 67: p. 14-38.

39. Liu, B. and Y.-K. Liu, Expected value of fuzzy variable and fuzzy expected value models. IEEE transactions on Fuzzy Systems, 2002. 10(4): p. 445-450.

40. Zhu, H. and J. Zhang. A credibility-based fuzzy programming model for APP problem. in Artificial Intelligence and Computational Intelligence, 2009. AICI'09. International Conference on. 2009. IEEE.

41. Pishvaee, M.S., J. Razmi, and S.A. Torabi, Robust possibilistic programming for socially responsible supply chain network design: A new approach. Fuzzy sets and systems, 2012. 206: p. $1-20$.

42. Liu, B. and K. Iwamura, Chance constrained programming with fuzzy parameters. Fuzzy sets and systems, 1998. 94(2): p. 227-237. 
43. Liu, B., Dependent-chance programming with fuzzy decisions. IEEE Transactions on Fuzzy Systems, 1999. 7(3): p. 354-360.

44. Davis, P. and T. Ray, A branch-bound algorithm for the capacitated facilities location problem. Naval Research Logistics (NRL), 1969. 16(3): p. 331-344.

45. Mirchandani, P.B. and R.L. Francis, Discrete location theory. 1990.

46. Benders, J.F., Partitioning procedures for solving mixed-variables programming problems. Numerische mathematik, 1962. 4(1): p. 238-252.

47. Geoffrion, A.M. and G.W. Graves, Multicommodity distribution system design by Benders decomposition. Management science, 1974. 20(5): p. 822-844.

48. Rei, W., et al., Accelerating Benders decomposition by local branching. INFORMS Journal on Computing, 2009. 21(2): p. 333-345.

49. Fischetti, M. and A. Lodi, Local branching. Mathematical programming, 2003. 98(1-3): p. 23-47.

50. Hamming, R.W., Error detecting and error correcting codes. Bell Labs Technical Journal, 1950. 29(2): p. 147-160.

51. Azadeh, A., R. Babazadeh, and S. Asadzadeh, Optimum estimation and forecasting of renewable energy consumption by artificial neural networks. Renewable and Sustainable Energy Reviews, 2013. 27: p. 605-612.

52. van Eijck, J., et al., Global experience with jatropha cultivation for bioenergy: an assessment of socio-economic and environmental aspects. Renewable and Sustainable Energy Reviews, 2014. 32: p. 869-889. 